\title{
Redundant actuation system of an underwater vehicle
}

\author{
B. Ropars ${ }^{1}$, L. Lapierre*, A. Lasbouygues ${ }^{* *, 2}$, D. Andreu ${ }^{* * *}$, R. Zapata**** \\ LIRMM Institute, University of Montpellier, 161 Rue Ada, Montpellier, France
}

\section{ARTICLE INFO}

\section{Keywords:}

Robotics

Underwater

Redundancy

\begin{abstract}
A B S T R A C T
This paper addresses the question of redundancy management of a vectorial actuation system of an underwater robot. The approach focuses on the compensation of the actuators structural imperfections: dead-zone and motor's response disparity. The solution is based on the identification of a correction matrix which highly improves the open-loop response of the actuation system. The effect is formally shown in the linear case approximation. Experimental validation shows the performance of the solution.
\end{abstract}

\section{Introduction}

The harsh condition of the underwater environment imposes difficult constraints to be handled by the robotic system. Moreover, recent applications require reactivity, robustness and dependability of the actuation system, as the survey paper (Johansen and Fossen, 2013) clearly underlines.

Major issues refer to the design and management of the system's actuators. Indeed, actuation redundancy plays an instrumental role on these questions. A large number of underwater vehicles use redundant actuation configuration, as, for example, "KAIKO 7000" (Nakajoh et al., 2012) of JAMSTEC or the "ODIN" (Choi et al., 1994) of the University of Hawaii without being exhaustive. Generally speaking, if the underwater vehicle holds more actuators than the 6 Degrees Of Freedom (DOF), the system is considered as over-actuated. Nevertheless, from the geometrical configuration of the thrusters depends the manageability of this redundancy (Fossen, 1994; Garus, 2004). Omerdic and Roberts (2004) addresses the structural redundancy to afford the system with fault tolerance in order to cope with the loss of a thruster. Fuqiang et al. (2013) focuses on the question of homing problem, in the case where the system has lost some of its actuation capability.

The structural redundancy of the system provides the ability to control several tasks, as the task function approach (Mansard, 2006) proposes. For example in Garus (2004), the authors use a configuration matrix of the actuation, allowing for actuators selection according to a secondary function related to dependability ability.

In Hanai et al. (2004) and Hanai et al. (2003) the authors propose to exploit redundancy following a heuristic that minimizes energy, despite a lack of compensation for motor nonlinearities. Chyba et al. (2008) uses an heuristic approach to optimize thrust efficiency.

A good characterization of thruster is needed to design realistic model of the thruster (Pivano, 2008), deals with the identification and design of underwater thruster. Kim et al. (2006) illustrates the difference between a closed loop control and open loop control, and shows that a good thrusters models is often sufficient for open-loop simple movement regulation, where no sensors are required.

Yoerger et al. (1990) and Bessa et al. (2013) address the influence of thruster dynamics on the behavior of the underwater vehicles. The authors propose a series of corrective filters to decrease the effect of nonlinearities, and those caused by a deterioration of a thruster performance. They show that these effects can cause not only a loss of performance, but also a limit cycle in response to control for dynamic positioning.

The redundancy management is an important theme of humanoid robotics, or in the domains of manipulation Mansard (2006) and Khalil et al. (2003). The task function approach is used in Flacco et al. (2012) to avoid singularities and saturation of the actuators. Nakamura et al. (1987) addresses the priority that can be attributed to secondary tasks for robot manipulators.

\footnotetext{
* Corresponding author.

** Corresponding author.

*** Corresponding author.

***** Corresponding author.

Email addresses: ropars@lirmm.fr (B. Ropars); lapierre@lirmm.fr (L. Lapierre); lasbouygues@lirmm.fr (A. Lasbouygues); andreu@lirmm.fr (D. Andreu); zapata@lirmm.fr (R. Zapata)

1 B. Ropars is working both at LIRMM Institute and CISCREA Company (www.ciscrea.net).

2 A. Lasbouygues work is supported by the Labex NUMEV.
} 
In our case, we use the task function approach to concurrently consider additional constraints on the actuators of a redundant underwater vehicle, in order to remove the effects of thrusters dead zones, parametric uncertainty, to respect the actuators saturation, and to manage the actuation reactivity. For previous work and a more complete bibliography on the subject, please refer to Ropars et al. (2015) and the references therein.

The paper is organized as follows. Section 2 introduces the notation, problem statement and presents the system that is used as study case and on which experimental validation is performed. The effects of actuators dead-zone and actuators' characteristic disparity is shown and analyzed. Section 3 exposes our proposition. This solution is analyzed in section 4, and experimental validation is rerouted at Section 5. Section 6 concludes this study and presents the perspective it opens.

\section{Problem statement}

This study is based on the Remotely Operated Vehicle (ROV), Jack, manufactured by the Ciscrea Company, on which electronic and control architectures has been rebuilt. Fig. 1 shows the system, carrying 6 thrusters able to induce actions on the three components of translation (surge, sway and heave) and two components of rotation (roll and yaw). Only the pitch is not controllable, but naturally stabilized because the buoyancy center is placed over the center of mass (this restoring torque effect is also present in roll).

This study tackles the question of redundancy management of actuation system of Jack and particularly the horizontal actuation system because our robot has 4 thrusters to control 3 degrees of freedom (surge, sway, yaw). As it will be shown in the sequel, the redundancy management will allow for reducing the effect of motors' characteristic disparity, as well as dead-zone effects.

\subsection{Notation}

In the sequel, 'bold' notation is reserved for vectors and matrices, while scalars are written classically. Let $\{U\}$ be the universal coordinate frame and $\{B\}$ be the body frame. In the sequel, $\boldsymbol{\eta}_{U}$ expresses the system state in $\{U\}, \boldsymbol{\nu}_{B}$ is the system velocities in $\{B\}$ and $\mathbf{F}_{B}$ represents forces and torques (provided by the actuation system) w.r.t. $\{B\}$, as

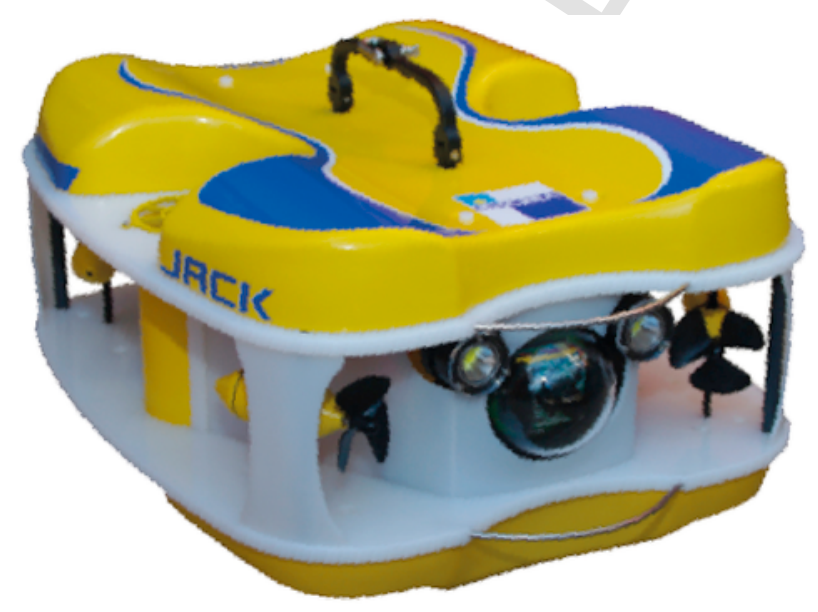

Fig. 1. The Jack system. stated on Equation (1) and illustrated at Fig. 2.

$$
\begin{array}{ccc}
\eta_{U}= & {[x, y, z, \phi, \theta, \psi]^{T}} \\
v_{B}= & {[u, v, w, p, q, r]^{T}} \\
\mathbf{F}_{B}= & {\left[F_{u}, F_{v}, F_{w}, \Gamma_{p}, \Gamma_{q}, \Gamma_{r}\right]^{T}}
\end{array}
$$

Referring to Fig. 2, each of the 4 horizontal actuators (motor + propeller) is mounted on the system with positioning parameters defined w.r.t $\{B\}$, for motor $i$, defined as the set $\left[d_{x, i}, d_{y, i}, d_{z, i}, \theta_{m, i} \psi_{m, i}\right]^{T}$.

The system kinematic model is classically taken as:

$$
\dot{\eta}_{u}=\mathbf{R}(\phi, \theta, \psi) \cdot v_{B}
$$

where $\mathbf{R}(\phi, \theta, \psi)$ denotes the kinematic relation between velocities expressed in the body frame $\{B\}$ and universal frame $\{U\}$, using Euler angle $(\phi, \theta, \psi)$.

$\mathbf{R}(\phi, \theta, \psi)$

$=\left[\begin{array}{ccr}\cos \psi \cos \theta & \cos \psi \sin \theta \sin \phi-\sin \psi \cos \phi & \sin \psi \sin \phi+c \\ \sin \psi \cos \theta & \sin \psi \sin \theta \sin \phi+\cos \psi \cos \phi & \sin \psi \sin \theta \cos \\ -\sin \theta & \cos \theta \sin \phi & \cos \psi \\ 0 & 0 & \\ 0 & 0 & \\ 0 & 0 & \end{array}\right.$

The system dynamic model structure is classically considered as exposed in Equation (4).

$$
\begin{array}{llll}
F_{u} & = & X_{\dot{u}} \cdot \dot{u}+X_{u \cdot|u|} \cdot u \cdot|u|+X_{w \cdot q} \cdot w \cdot q+X_{v \cdot r} \cdot v \cdot i \\
F_{v} & = & Y_{\dot{v}} \cdot \dot{v}+Y_{v \cdot|v|} \cdot v \cdot|v|+Y_{u \cdot r} \cdot u \cdot r+Y_{w \cdot p} \cdot w \cdot p \\
F_{w} & = & Z_{\dot{w}} \cdot \dot{w}+Z_{w \cdot|w|} \cdot w \cdot|w|+Z_{u \cdot q} \cdot u \cdot q+Z_{v \cdot p} \cdot v \\
\Gamma_{p} & = & K_{\dot{p}} \cdot \dot{p}+K_{p \cdot|p|} \cdot p \cdot|p|+K_{q \cdot r} \cdot q \cdot r+K_{v} \cdot v+K_{w} \\
\Gamma_{q} & = & M_{\dot{q}} \cdot \dot{q}+M_{q \cdot|q|} \cdot q \cdot|q|+M_{p \cdot r} \cdot p \cdot r+M_{u} \cdot u+M_{w} \\
\Gamma_{r} & = & N_{\dot{r}} \cdot \dot{r}+N_{r \cdot|r|} \cdot r \cdot|r|+N_{p \cdot q} \cdot p \cdot q+N_{u} \cdot u+N_{v}
\end{array} .
$$

The hydrodynamic parameters $\left.\left[X_{(\cdot)}, Y_{(\cdot)}, Z_{(}\right), K_{(}\right), M_{(\cdot)}, N_{(\cdot)}$ are estimated using classic approach. In the study of autonomous vehicles, many works focuse on the identification of nonlinear dynamic model as in Yang et al. (2014), Low (2009), Indiveri (1998), Ridao et al. (2004), Avila et al. (2012), Liang et al. (2013). Yang et al. (2015) focuses on the modeling for the robot Jack, developed by CISCREA company, ${ }^{3}$ which is used as case study. Jack's model can be found in Yang et al. (2014) and Yang et al. (2015).

\subsection{Motor characteristic identification}

Each of these 4 actuators is controlled with input $c_{m, i}$ (expressed in terms of PWM) and results in a force $F_{m, i}$. The 4 actuators inputs are written as $(4 \times 1)$ vector $\mathbf{c}_{m}(5)$.

$\mathbf{c}_{m}=\left[c_{m, 1}, c_{m, 2}, c_{m, 3}, c_{m, 4}\right]^{T}$

3 www.ciscrea.net. 


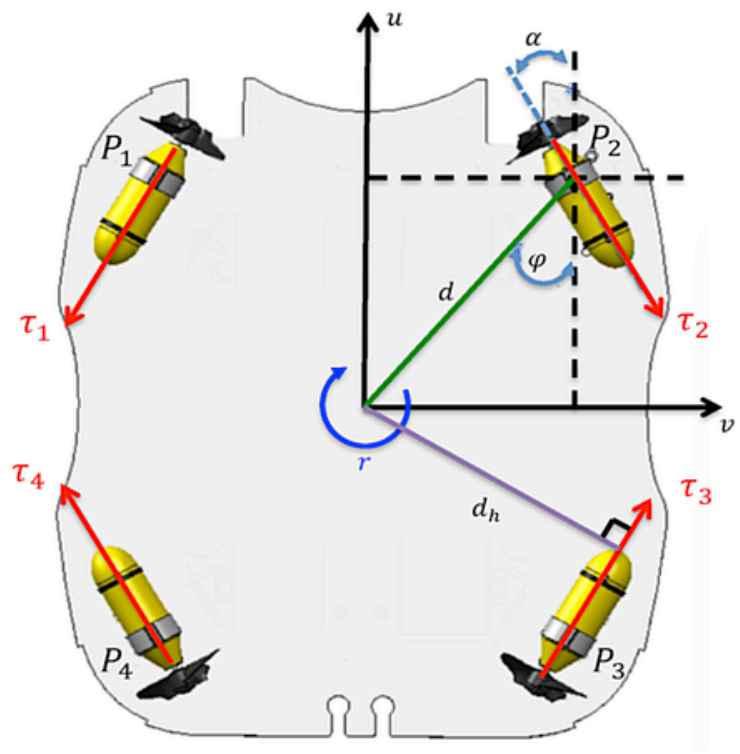

$\{B\}$

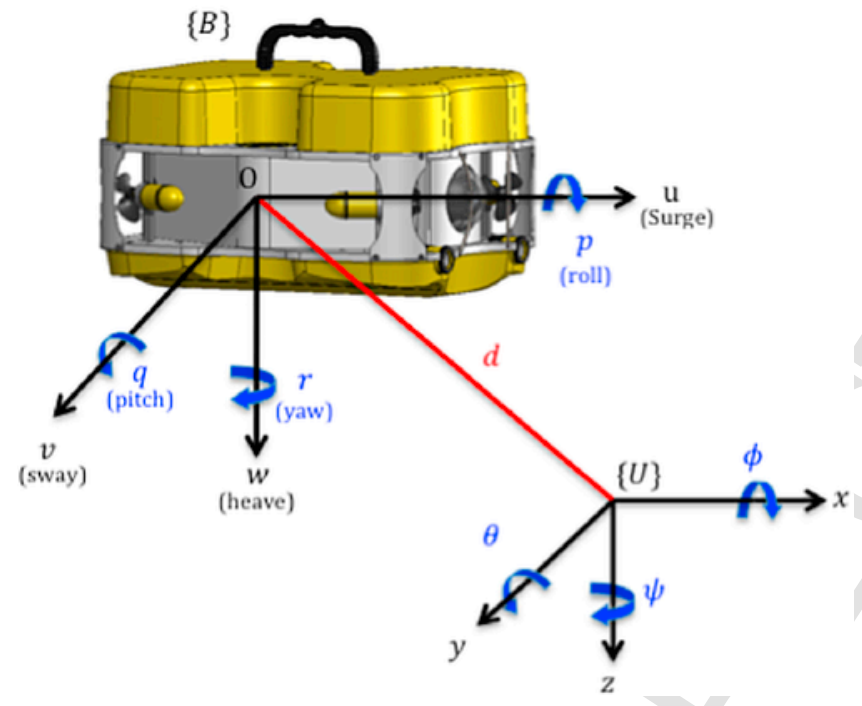

Fig. 2. Notation and frames definition, for Jack.

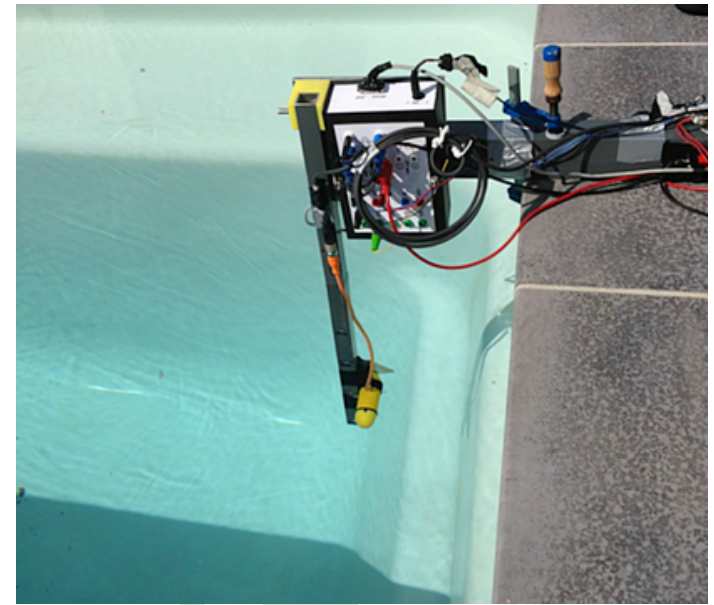

Fig. 3. Notation and frames definition.

The 4 actuators produce 4 forces written with the $(4 \times 1)$ vector $\mathbf{F}_{m}$ (6).

$\mathbf{F}_{m}=\left[F_{m, 1}, F_{m, 2}, F_{m, 3}, F_{m, 4}\right]^{T}$

The characteristic of one motor has been experimentally identified using a force-sensor mounted on the test bench shown at Fig. 3. The bench allows to realize an automatic procedure of identical forward and backward sequences to measure the force on each thruster. This is a static test where system velocity is not considered. Nevertheless, this method induces important improvement on open-loop system control as it will be experimentally shown in the sequel.

The actuator input/output relation is denoted $F_{m, i}=\mathrm{M}_{i}\left(c_{m, i}\right)$, for actuator $i$.

Hence, we have covered the full range of motor inputs ( $\left.c_{m, i} \in[-100 ; 100]\right)$ and measured the resulting force $F_{m, i}$. The result is given at Fig. 4.

Analysing the actuator response of Fig. 4, we extract the following characteristics:

- An important dead-zone is present in the motor response. $\{B\}$ We define as $\left[c_{m}^{D Z-}, c_{m}^{D Z+}\right]$ the set of actuation inputs which does not induce any thrust (neither motor rotation). Hence the minimal attainable positive thrust is denoted $F_{m}^{D Z+}$, and the maximal negative thrust is denoted $F_{m}^{D Z-}$.

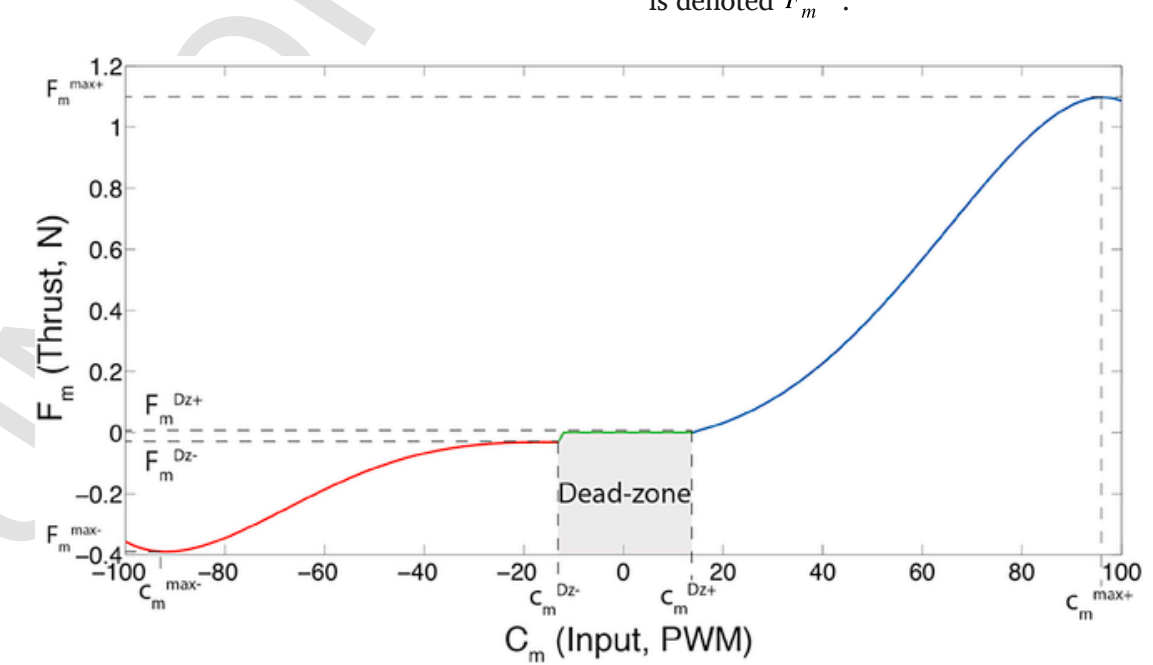

Fig. 4. Motor characteristic identification. 
- the maximum positive thrust is denoted as $F_{m}^{\max +}$, the minimum one is denoted $F_{m}^{\max -}$.

Hence, we define an augmented set of actuator parameters, which includes the characteristic points previously identified. Hence for the motor $i$ :

$\mathbf{P}_{m, i}=\left[d_{x, i}, d_{y, i}, d_{z, i}, \psi_{m, i}, \theta_{m, i}, c_{m, i}^{D Z-}, c_{m, i}^{D Z+}, F_{m, i}^{\max -}, F_{m, i}^{\max +}\right]^{T}$

\subsection{Actuation system}

Each (theoretically identical) thruster $i$ is mounted symmetrically, as shown at Fig. 2, and produces its force $F_{m, i}$ with angles $\psi_{m, i}$ w.r.t $u$ axis of $\{B\}$. The inertial torque generated by the motor rotation is neglected, considering the facilitating effect of a symmetrical distribution of direct and reverse propellers that globally nullifies the common regime of actuators inertia effect. Hence, the resulting effect of the actuation system is the set of 3 forces and torque $\mathbf{F}_{B}$, expressed in $\{B\}$ as:

$\mathbf{F}_{B}=\left[F_{u}, F_{v}, \Gamma_{r}\right]^{T}$

The relation between $\mathbf{F}_{B}$, and $\mathbf{F}_{m}$, is expressed thanks to the constant matrix A (9).

$\mathbf{F}_{B}=\mathbf{A} \cdot \mathbf{F}_{m}$

where $\mathbf{A}$ is a $(3 \times 4)$ matrix, function of the motor positions $\left[d_{x, i}, d_{y, i}, d_{z, i}, \psi_{m, i}\right]^{T}$, for $i=1, \ldots, 4$.

\subsection{Redundancy management}

The matrix $\mathbf{A}$ is not square and expresses the system redundancy. We verify that $\mathbf{A}$ is full-rank, expressing the fact that the 3 DoFs of $\mathbf{F}_{B}$ are controllable through the actuation system. The control design requires the inversion of the relation (9), facing the redundancy question. Among all possible solutions, the classic approach uses the Moore-Penrose pseudo-inverse matrix denoted $\mathbf{A}^{+}$, computed according to the relation (10), if $A \cdot A^{T}$ is invertible, which is the case if singular values of $A$ are different from zero. This is linked with the effectiveness of system redundancy. In our case, where we have more thrusters than DoF and where they are geometrically installed with a vectorial configuration, the invertibility of $A \cdot A^{T}$ is warranted.

$$
\mathbf{A}^{+}=\mathbf{A}^{T} \cdot\left(\mathbf{A} \cdot \mathbf{A}^{T}\right)^{-1}
$$

Then, the inverse actuation relation is computed according to equation (11)

$$
\mathbf{F}_{m}=\mathbf{A}^{+} \cdot \mathbf{F}_{B}^{d}
$$

where $\mathbf{F}_{B}^{d}$ denotes the desired resulting action, expressed in the body frame $\{B\} . \mathbf{F}_{B}^{d}$ can be seen as the output of a control law, or an operator intention.

As stated in Golub and Van Loan (1957) and Horn and Johnson (1985), the use of the Moore-Penrose pseudo inverse $\mathbf{A}^{+}$brings a solution $\mathbf{F}_{m}$ with minimum norm, i.e. energetically optimal. Moreover, con- sidering (9), the induced body-frame action is:

$\mathbf{F}_{B}=\mathbf{A} \cdot \mathbf{F}_{m}=\mathbf{A} \cdot \mathbf{A}^{+} \cdot \mathbf{F}_{B}^{d}$

where $F_{B}^{d}$ stands for the actuation demand (coming from operator or control structure), and $F_{B}$ denotes the effective action induced by the actuation system. Note that, as stated in Horn and Johnson (1985), if singular values of $\mathbf{A}$ are different from $0, \mathbf{A} \cdot \mathbf{A}^{+}=\mathbb{1}_{3}$, where $\mathbb{1}_{3}$ denotes the $(3 \times 3)$ identity matrix.

The system redundancy can be exploited using the kernel-projector $\mathbb{P}_{\text {Krnl }}=\left(\mathbb{Q}_{4}-\mathbf{A}^{+} \cdot \mathbf{A}\right)$, as originally stated by A. Ligeois, in 1977 (Liégeois, 1977). He showed that:

$$
\mathbf{F}_{m}=\mathbf{A}^{+} \cdot \mathbf{F}_{B}^{d}+\mathbb{P}_{K r n l} \cdot \mathbf{X}
$$

implies

$$
\mathbf{F}_{B}=\mathbf{A} \cdot \mathbf{A}^{+} \cdot \mathbf{F}_{B}^{d}+\underbrace{\left(\mathbf{A}-\mathbf{A} \cdot \mathbf{A}^{+} \cdot \mathbf{A}\right)}_{=0} \cdot \mathbf{X}=\mathbf{A} \cdot \mathbf{A}^{+} \cdot \mathbf{F}_{B}^{d}
$$

since (10) and for any $(4 \times 1)$ vector $\mathbf{X}$. Following the same idea, consider now a $(4 \times 1)$ matrix $\mathbf{M}_{m}$ such that

$$
\mathbf{M}_{m} \in \operatorname{ker}(\mathbf{A}) \Rightarrow \mathbf{A} \cdot \mathbf{M}_{m}=0
$$

Then, considering the previous result, one can state that if:

$$
\mathbf{F}_{m}=\mathbf{A}^{+} \cdot \mathbf{F}_{B}^{d}+\mathbf{M}_{m} \cdot r_{m}
$$

or, a compact form:

$\mathbf{F}_{m}=\left[\mathbf{A}^{+} \mathbf{M}_{m}\right] \cdot\left[\begin{array}{l}\mathbf{F}_{B}^{d} \\ r_{m}\end{array}\right]$

then (12) is true, for any vector $r_{m}$, since (15).

The redundancy is manageable through the choice of $\mathbf{M}_{m}$, respecting $\mathbf{M}_{m} \in \operatorname{ker}(\mathbf{A})$ and $r_{m}$

\subsection{Case study}

Recall that we restrict this study to the 4 horizontal thrusters of the Jack robot. Considering the (nominal) parameters value as:

$$
\begin{aligned}
x_{m} & =.21 m \\
y_{m} & =.14 m \\
z_{m} & =.2 m \\
\psi_{m} & =\frac{\pi}{6}
\end{aligned}
$$

the A matrix is then computed:

$$
\mathbf{A}=\left[\begin{array}{cccc}
-.866 & -.866 & .866 & .866 \\
-.5 & .5 & .5 & -.5 \\
.226 & -.226 & .226 & -.226
\end{array}\right]
$$


We propose the following candidate for $\mathbf{M}_{m}$ :

$\mathbf{M}_{m}=[1,1,1,1]^{T}$

One can easily verify that, on our system, $\mathbf{M}_{m} \in k e r(\mathbf{A})$. This illustrates the interesting property, brought by the vectorial configuration of the thrusters, stating that: an identical action of the actuators results in a null action w.r.t. $\{B\}$.

In Ropars et al. (2015), we use this idea to compensate the actuator dead-zone effect. We experimentally showed the undesired effect of the presence of dead-zone in the actuators' characteristics, which produces oscillatory behavior of the system or introduces an offset error in system response, despite a yaw control loop. This experimentation has been conducted on the Jack system (cf. Fig. 1). The results are shown at Fig. 5. The dead zone compensation has been considered in three ways:

- without compensation, the system response presents an offset, corresponding to the dead-zone limitation, in blue on Fig. 5.

- Contraction of the dead-zone: the actuators inputs corresponding to the identified dead-zone have been removed from accessible inputs. The consequence is the apparition of an oscillatory behavior, inducing unnecessary motor fatigue, and a response performance dependent of the motor reactivity. Results are shown in red on Fig. 5.

- Compensation using a common motor regime, applied to all the horizontal actuators. Results are shown in black and indicates the removal of the offset and the chatterring effect.

Nevertheless, another consequence of motors' characteristic disparity, which is not shown in results of Fig. 5 is the occurrence of unde- sired linear movement, caused by the difference between each actuators characteristic. Indeed, the closed loop control of the yaw angle compensates for the undesired rotational movements, but the linear ones are not compensated. This could be done using the same principle by considering $(u, v, w)$ control laws to regulate the linear velocities to 0 . But this requires the use of a DVL sensor, expensive and cumbersome, i.e. unadapted for small and low cost underwater system.

The object of the study (Ropars et al., 2015) was to investigate the redundancy that the Jack system offers, concerning the motor regime management in order to avoid problematic effects as dead-zone, saturation and lack of reactivity. In the present paper, we investigate the effects of actuators characteristic disparity, and propose a solution to reduce them.

But, first of all, we have to analyze deeper the effect of the actuators characteristic disparity on the system response.

\subsection{Actuators' response disparity}

If all the actuators share the same characteristic, an identical motor input produces null resulting forces and torques in $\{B\}$. Of course, the actuator responses are never perfectly identical. This actuators' response disparity induces undesired effects as experimentally noticed, reported at Fig. 16.

In order to investigate this question, we have developed a simulator that considers different actuators' response, and reproduces the problematic coupling effect. As a generic profile for the actuator response established from Fig. 4, we consider the expression of Equation (21)
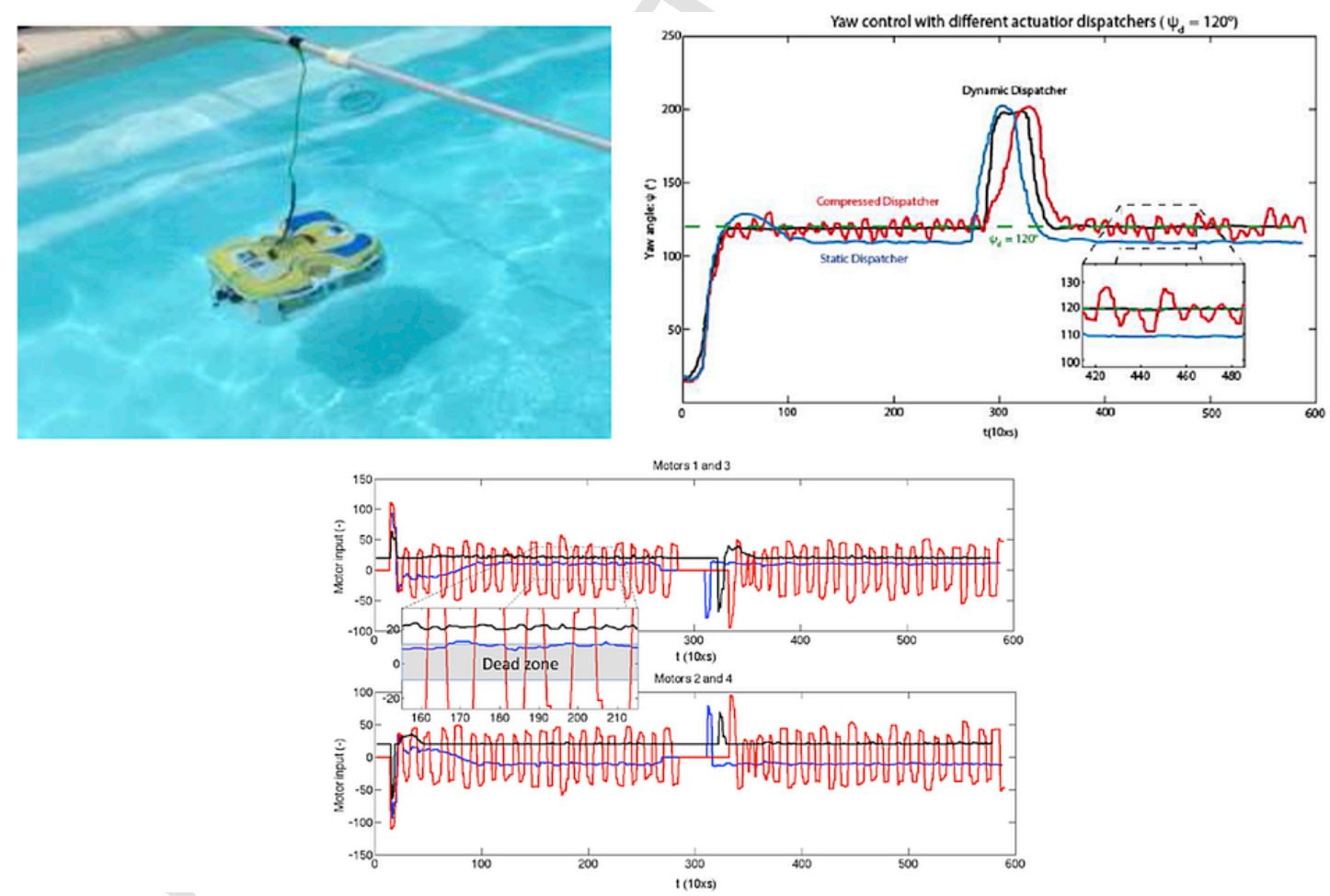

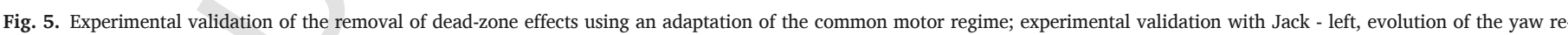

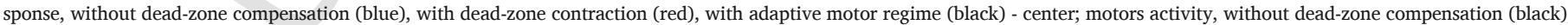

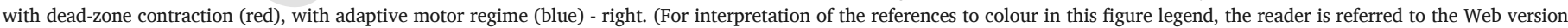
of this article.) 
which qualitatively describes the expected actuator's response.

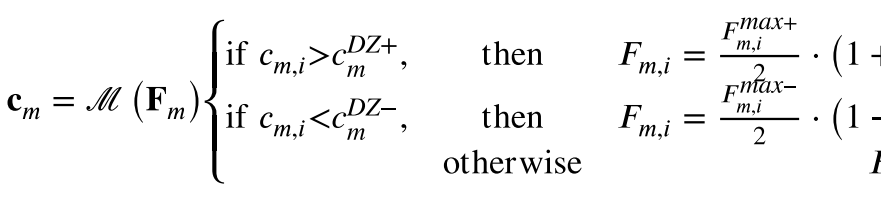

Actuators characteristics (21) parameters are chosen with a randomized valuation as exposed in Table 1 , where the randomization is of $n \%$ range, around nominal values defined at entry $\Lambda$ of Table 1 .

The parameters of Table 1 result in the actuator characteristics of Fig. 6.

Of course, we consider these characteristics as unknown. We also consider an estimated characteristic, identical for all actuators, corresponding to the nominal values expressed at Table 1 , with entry denoted $\Lambda$, and drawn in dashed-orange at Fig. 6 . Note that for control computation purpose, we need to have the inverse characteristics of this estimated profile. We obtain the analytical expression for the inverse estimated characteristic expressed at Equation (22).

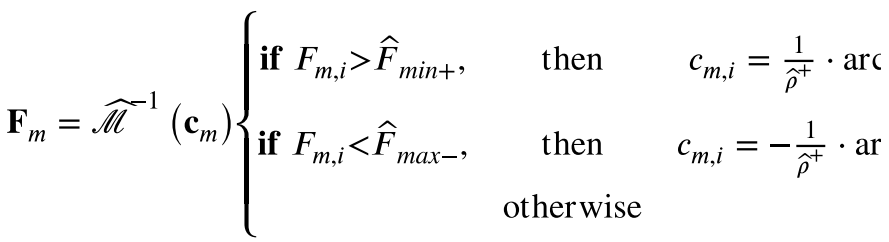

where

$$
\begin{aligned}
& \widehat{F}_{\text {min }+}=\frac{\hat{F}_{m}^{\text {max }}}{2} \cdot\left(1+\tanh \left(\hat{\rho}^{+} \cdot\left(\hat{c}_{m,}^{D Z+}-50\right)\right)\right) \\
& \widehat{F}_{\text {max }-}=\frac{\hat{F}_{m}^{\text {max }}}{2} \cdot\left(1-\tanh \left(\hat{\rho}^{-} \cdot\left(\hat{c}_{m,}^{D Z+}-50\right)\right)\right)
\end{aligned}
$$

and $\widehat{F}_{m}^{\max +}, \widehat{F}_{m}^{\max -}, \hat{\rho}^{+}, \hat{\rho}^{-}, \hat{c}_{m}^{D Z+}$ and $\hat{c}_{m}^{D Z-}$, are defined at Table 1 , with entry $\Lambda$.

The effects of this disparity is firstly illustrated using the same input for all the motor, $c_{0}=50 \mathrm{PWM}$, corresponding to a common regime $r_{m}=\widehat{\mathscr{M}}^{-1}\left(c_{0}\right)$ (which should induce no movement if motor's characteristics are the same for all actuators, as stated in (15)), cf. Fig. 7-up. Another consequence of this disparity is to produce an undesired coupling effect between the system degrees of freedom, cf. Fig. 7-down, where a desired $\mathbf{F}_{B}^{d}=[1,0,0]^{T}$ and $\mathbf{F}_{B}^{d}=[0,1,0]^{T}$ have been considered.

The vectorial configuration of the horizontal thrusters should theoretically induce the respect of condition (14), which is clearly not the case of the simulation results of Fig. 7-up. The actuators disparity induces undesired linear and rotational movements. This problematic behavior is, of course, also encountered in experimental conditions as it

Table 1

\begin{tabular}{|c|c|c|c|c|c|c|}
\hline & $c_{m}^{D Z-}$ & $c_{m}^{D Z+}$ & $F_{m, i}^{\max -}$ & $F_{m, i}^{\max +}$ & $\rho^{+}$ & $\rho^{-}$ \\
\hline$n \%$ & 20 & 20 & 100 & 100 & 10 & 10 \\
\hline 1 & -14.0460 & 16.2510 & -0.5946 & 1.0688 & 0.0513 & 0.0480 \\
\hline 2 & -14.2920 & 14.3580 & -0.6241 & 0.9694 & 0.0488 & 0.0523 \\
\hline 3 & -13.9365 & 15.7710 & -0.4753 & 0.5119 & 0.0500 & 0.0475 \\
\hline 4 & -13.9080 & 15.7605 & -0.2919 & 0.8371 & 0.0510 & 0.0514 \\
\hline$\wedge$ & -17 & 17 & -0.5 & 1 & 0.05 & 0.05 \\
\hline
\end{tabular}

Actuators parameters used in simulation. will be exposed in the sequel. The curves drawn at Fig. 7-down shows the undesired coupling effect between system's DoFs.

Concerning the compensation of rotational movements, the use of an Inertial Measurement Unit (IMU) allows for regulating the yaw angle with a classic PID control, whose action is simply added to the common motor regime $\left(r_{m}=\widehat{\mathscr{M}}^{-1}\left(c_{0}\right)\right)$, as illustrated with the upper curves of Fig. 8 and expressed at Equation (24). Note that this simple control works because of the regulation around 0 . We are not interested here in the controller performances, but, as we will see later, in the stationary values which the actuators inputs reach. Also note the importance of the integral action, which allows the compensation of thrusters' disparity, for the rotational movement.

$$
\left.\begin{array}{c}
\mathbf{F}_{m}=\mathbf{A}^{+} \cdot\left[\begin{array}{c}
0 \\
0
\end{array}\right. \\
1 \cdot(-\psi)+1 \cdot(-r)+0.1 \cdot \int_{0}^{t}(-\psi) \cdot d t
\end{array}\right]
$$

This strategy is generally used for compensating the actuation disparity effects on attitude (system's orientation). But remains the problematic linear movements. This effect can also be seen when an actuation demand is asked. Fig. 8-down shows the system response to $\mathbf{F}_{B}^{d}=[1,0,0]^{T}$ (bold lines) along the surge axis, and $\mathbf{F}_{B}^{d}=[1,0,0]^{T}$ (thin lines) along sway. It clearly shows that the heading regulation do not prevent the coupling effects of actuators' characteristic disparity.

The compensation of this undesired effect can be similarly done if a linear velocity measurement is available. Doppler Velocity Log (DVL) can be advantageously used in such a situation, but this type of system is expensive and cumbersome, unadapted for small vehicle, which is our case. The purpose of the next chapter is to propose a method which allows for equilibrating the actuators action, in order to reduce this undesired effect without using DVL.

\section{Actuators disparity compensation}

The method is based on a closed loop identification of corrective coefficients to apply to motor inputs. This on-line identification is done using an IMU for attitude regulation, and possibly a DVL or a vision system. The drawbacks of these solution are:

- DVL sensors are expensive and cumbersome for small-size underwater systems. Its has a significant impact on the static and dynamic properties of the system, which may require to increase the system actuation capabilities (power and reactivity).

- A vision system can be used in this context, with a position or optical flow visual servoing, but requires a low water turbidity.

First, we illustrate the method using simulation, where any kind of measurement is easy to obtain. In a second time, we experimentally validate our approach. 


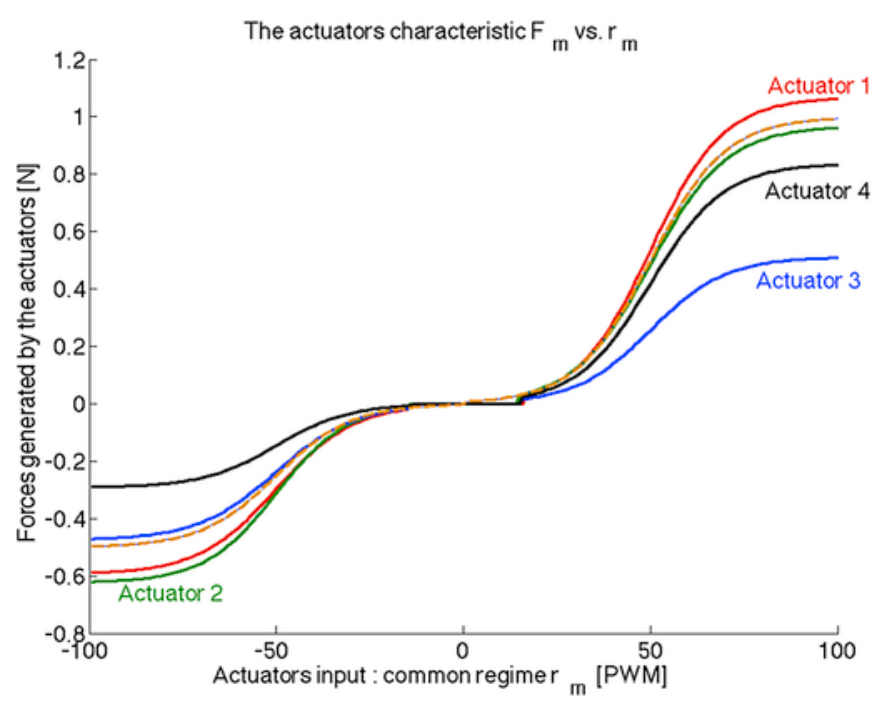

Fig. 6. Illustration of the actuators' characteristics disparity (simulation).

\subsection{Identification of corrective actuator coefficients}

First, we identify corrective coefficients on each actuator in function of different common motor regimes, using PID controllers on each system axis. The control law is expressed at Equation (25). Once again, this control is valid only because the regulation is around 0 . Simulation results are shown at Fig. 9.

$$
\mathbf{F}_{m}=\mathbf{A}^{+} \cdot\left[\begin{array}{c}
1 \cdot(-u)+1 \cdot \int_{0}^{t}(-u) \cdot d t \\
1 \cdot(-v)+1 \cdot \int_{0}^{t}(-v) \cdot d t \\
1 \cdot(-\psi)+1 \cdot(-r)+0.1 \cdot \int_{0}^{t}(-\psi) \cdot d t \\
\mathbf{c}_{m}=\widehat{M}^{-1}\left(\mathbf{F}_{m}\right)+\mathbf{M}_{m} \cdot r_{m}
\end{array}\right]
$$

From the results of Fig. 9, we extract the stationary value that actuators inputs have reached. The corrective coefficients are computed according to

$\alpha_{i}\left(c_{0}\right)=\frac{c_{m, i}^{\infty}}{c_{0}}$

for $i=1,2,3,4$, and where $c_{m, i}^{\infty}$ denotes the static (final) value of the input of motor $i$. For the chosen common motor regime $c_{0}$, we build the $(4 \times 1)$ vector $\boldsymbol{\alpha}\left(c_{0}\right)$, as Equation (27) denotes.

$\alpha\left(c_{0}\right)=\left[\alpha_{1}\left(c_{0}\right), \alpha_{2}\left(c_{0}\right), \alpha_{3}\left(c_{0}\right), \alpha_{4}\left(c_{0}\right)\right]^{T}$

We proceed to the same test for different motor regimes ( $\left.c_{0} \in[-100 ; 100] \mathrm{PWM}\right)$, and plot the evolution of the components of $\alpha\left(c_{0}\right)$ in function of $c_{0}$. Results are drawn at Fig. 10 .

The analysis of Fig. 10 clearly shows that the range $c_{m, i} \in[-20,20]$ is not exploitable. From results of Fig. 10, we extract 4 functions $\alpha_{i}\left(c_{m, i}\right)$, composing the $(4 \times 4)$ diagonal matrix $\mathbb{Q}\left(\mathbf{c}_{m}\right)$, identified from
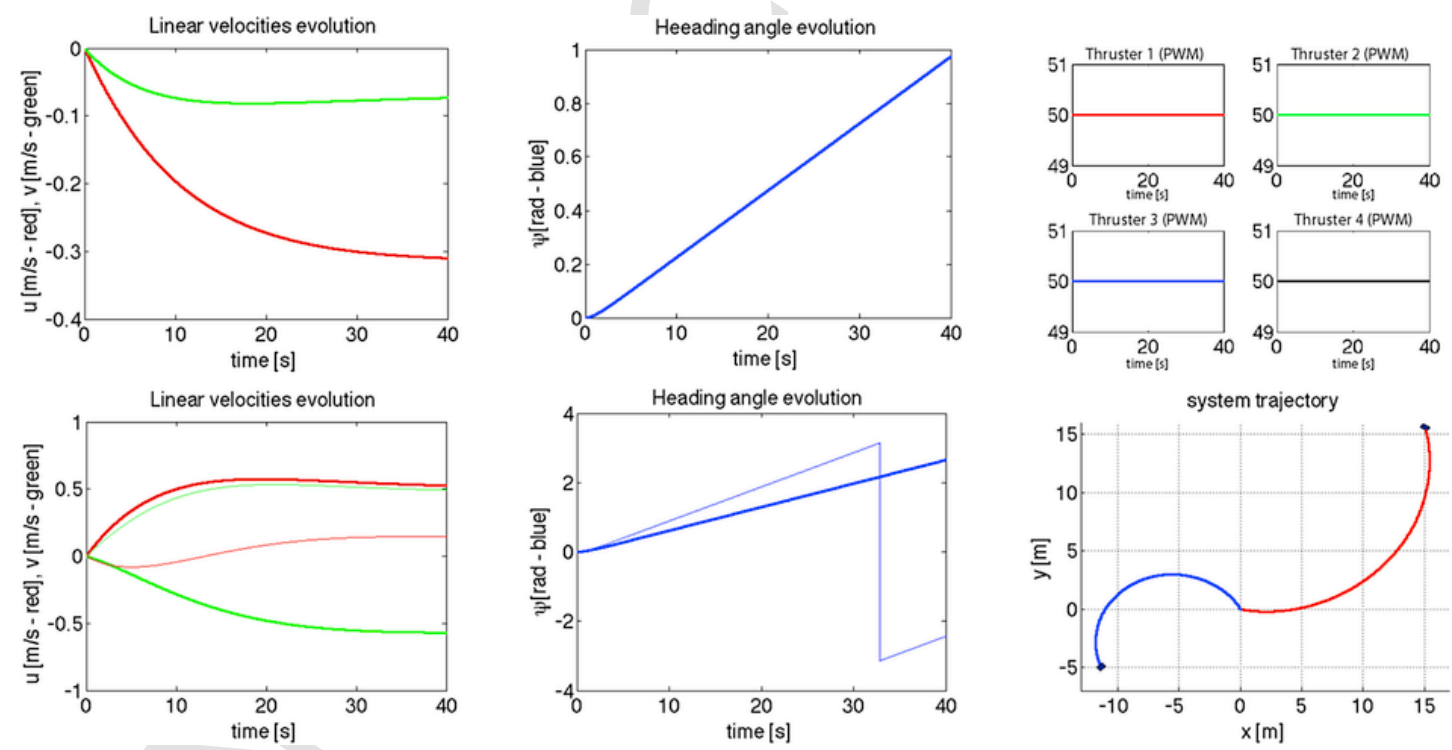

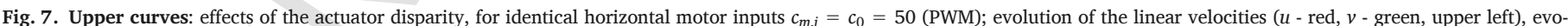

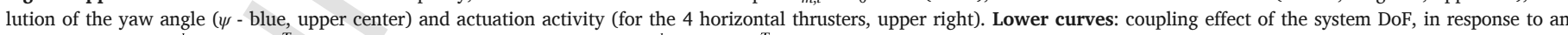

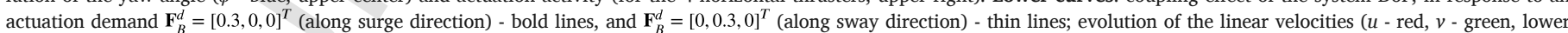

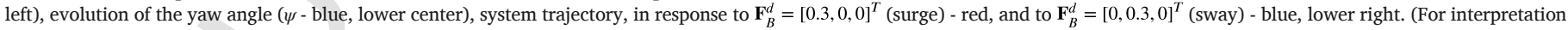
of the references to colour in this figure legend, the reader is referred to the Web version of this article.) 

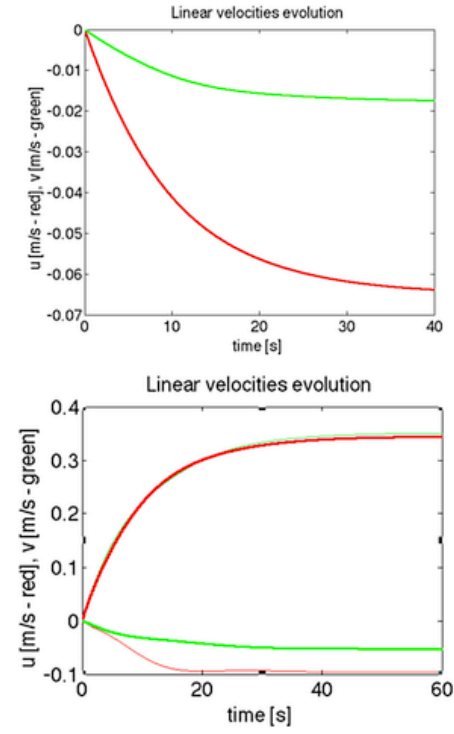
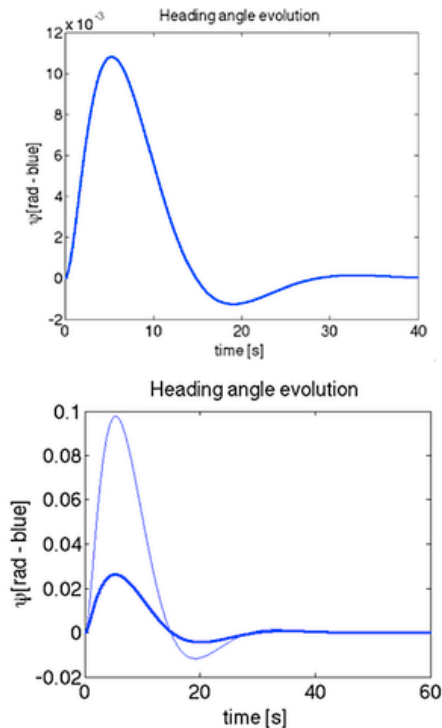
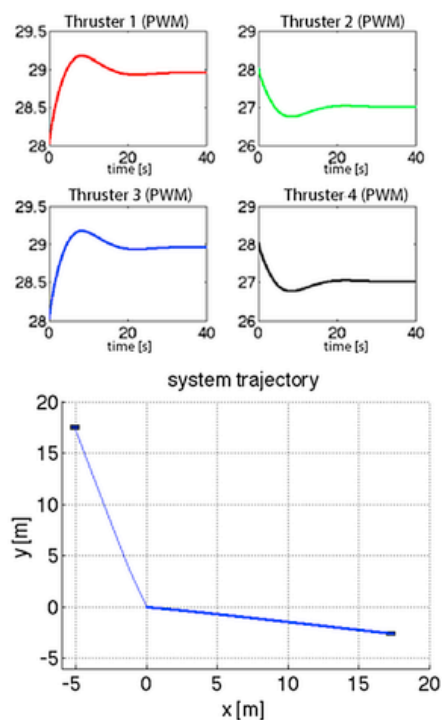

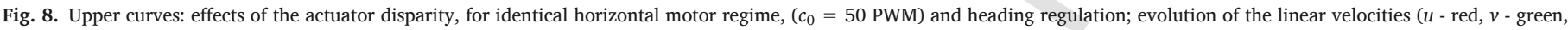

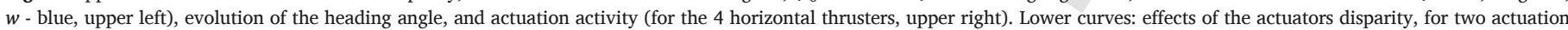

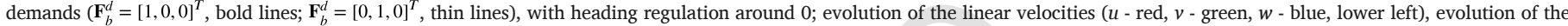

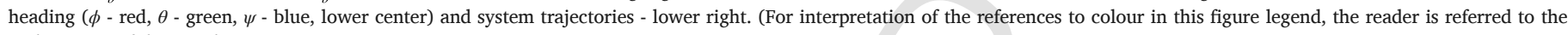
Web version of this article.)
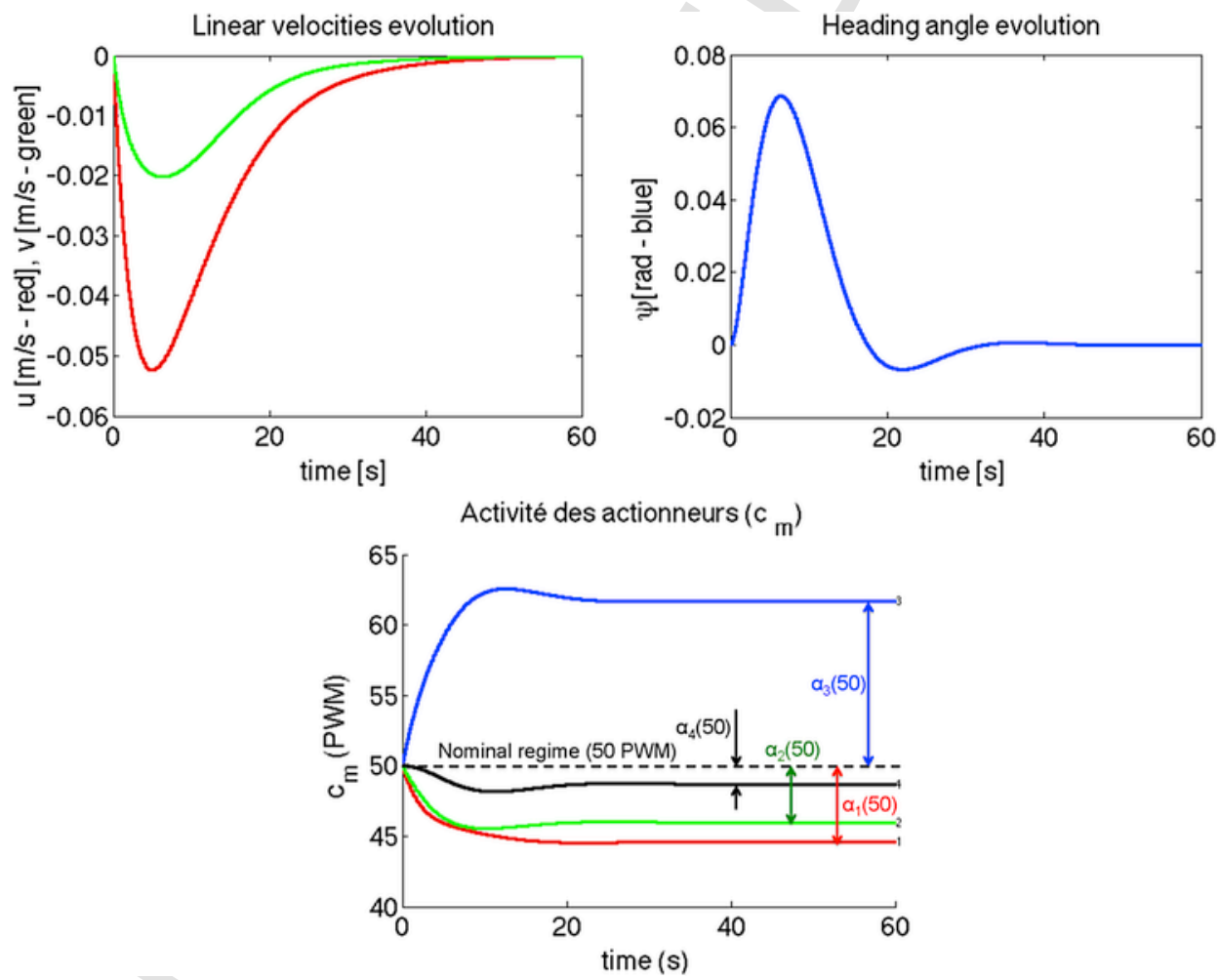

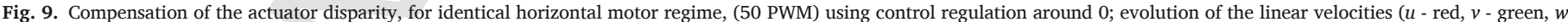

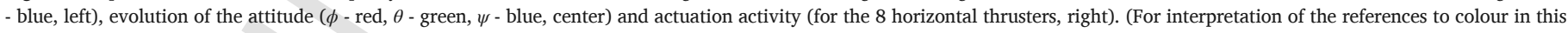
figure legend, the reader is referred to the Web version of this article.) 


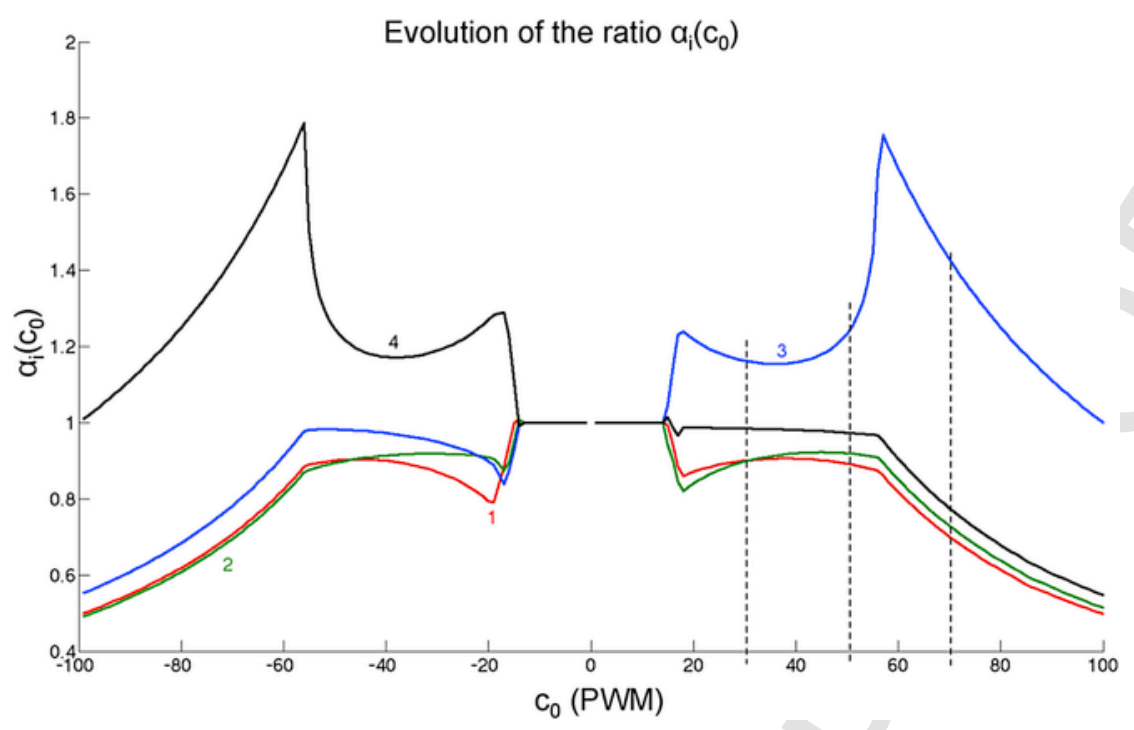

Fig. 10. Evolution of the corrective coefficient versus the common motor regime (simulation).

the curves drawn in Fig. 10.

$$
Q\left(\mathbf{c}_{m}\right)=\operatorname{diag}\left(\alpha_{1}\left(c_{m, 1}\right), \alpha_{2}\left(c_{m, 2}\right), \alpha_{3}\left(c_{m, 3}\right), \alpha_{4}\left(c_{m, 4}\right)\right)^{T}
$$

As a first illustration, let's consider the system, in open loop (without control), with a common motor regime chosen as $c_{0}=50 \mathrm{PWM}$, and apply to the actuator the following input:

$\mathbf{c}_{m}=Q\left(1_{4} \cdot c_{0}\right)^{T} \cdot 1_{4} \cdot c_{0}$
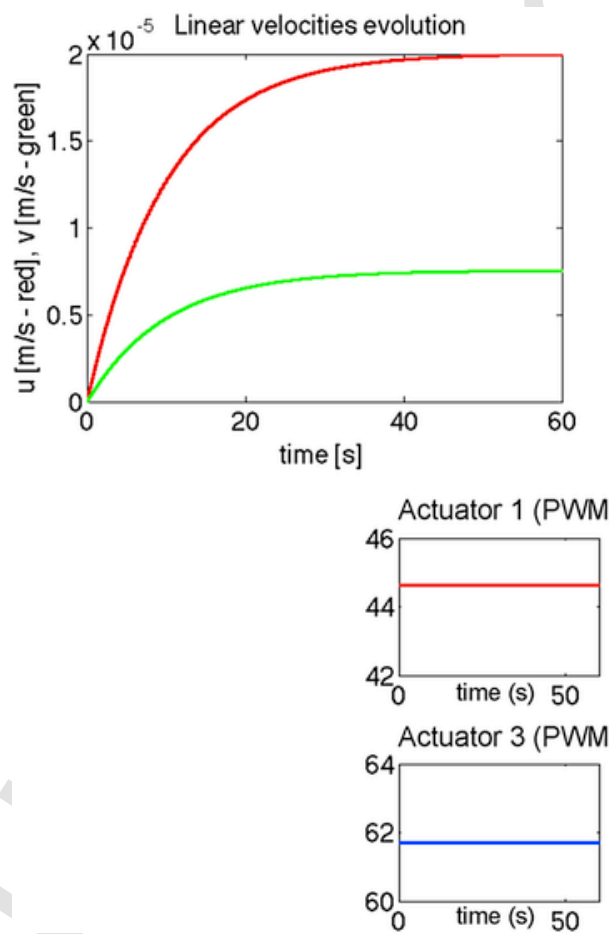

where the term $1_{4}$ stands for the $(4 \times 1)$ vector where each component is equal to $1,1_{4}=\left[\begin{array}{llll}1 & 1 & 1 & 1\end{array}\right]^{T}$. The results are drawn at Fig. 11 , to be compared to those obtained at Fig. 7-up. As expected, since the actuation input corresponds to the stationary regime of a stable solution, the system remains in the close vicinity of the origin. The compensation is done with the corrective matrix $\mathbb{Q}\left(1_{4} \cdot 50\right)$, as depicted at Fig. 10 .

We now generalize the use of the corrective coefficient to the situation where an actuation demand is present. We apply the following actuator input:
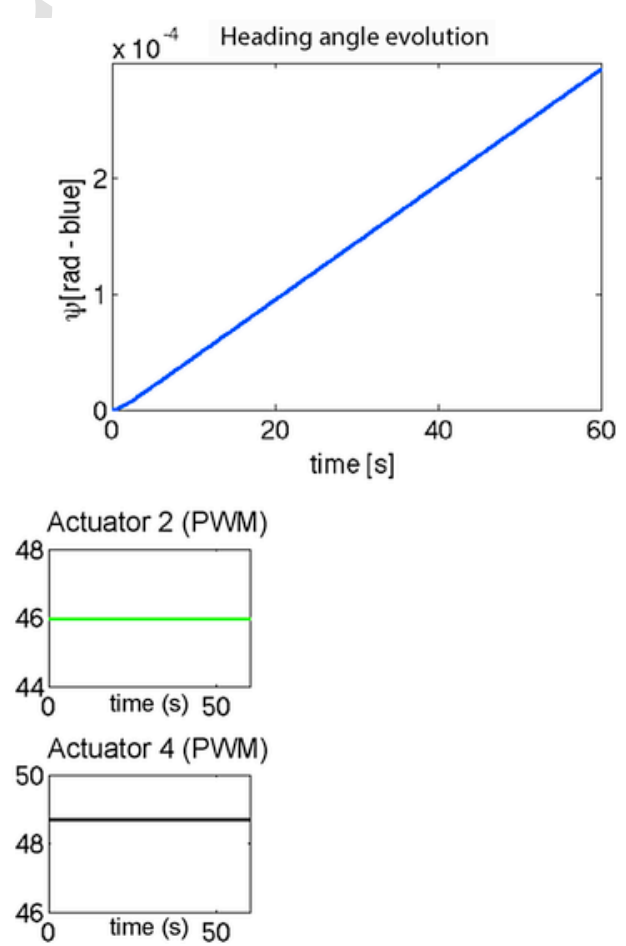

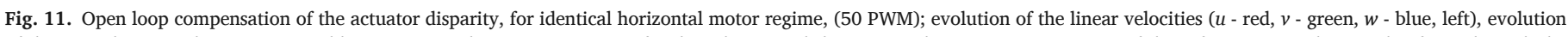

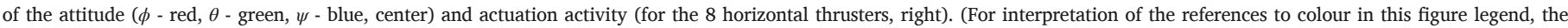
reader is referred to the Web version of this article.) 


$$
\mathbf{c}_{m}=\widehat{Q}\left(\widehat{\mathscr{M}}^{-1}\left(\mathbf{A}^{+} \cdot \mathbf{F}_{B}^{d}\right)+c_{0} \cdot 1_{4}\right)
$$

where, in the results shown at Fig. 12, the common regime is $c_{0}=50$ $\mathrm{PWM}$, and the actuation demand is $\mathbf{F}_{B}^{d}=[1,0,0]^{T}$ (along surge direction) - bold lines, and $\mathbf{F}_{B}^{d}=[0,1,0]^{T}$ (along sway direction) - thin lines. The results of Fig. 12 have to be compared to the lower curves of Fig. 7, clearly showing a great improvement of the system trajectories.

\section{Analysis}

The decoupling effect of Equation (30) can be formally explained within the linear approximation (if actuator's response is linear). It allows for a linear expression of the matrix M, diagonal and composed with the slope of the actuators' characteristic, illustrated at Fig. 13-left. The matrices $\mathrm{M}$ and $\widehat{\mathscr{M}}^{-1}$ are written at Equation (31).

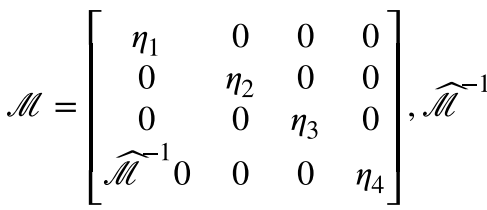

$$
\begin{aligned}
& =\left[\begin{array}{cccc}
\hat{\eta}^{-1} & 0 & 0 & 0 \\
0 & \hat{\eta}^{-1} & 0 & 0 \\
0 & 0 & \hat{\eta}^{-1} & 0 \\
0 & 0 & 0 & \hat{\eta}^{-1}
\end{array}\right]
\end{aligned}
$$

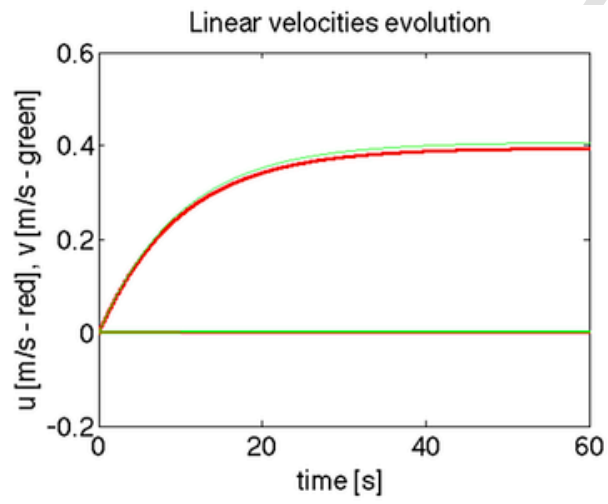

where $\eta_{\{1,2,3,4\}}$ denotes the (unknown) slope of actuators' characteristic, and $\hat{\eta}$ denotes the slope of the estimated characteristic. Hence,

$\mathbf{F}_{m}=\mathrm{M} \cdot \mathbf{c}_{m}$

Note that, when the inverse relation will be necessary for control computation, $\widehat{\mathscr{M}}^{-1}$ will be used instead of $\mathrm{M}^{-1}$.

For the simulation, we have chosen the following values: $\eta_{1}=1.06 \cdot 10^{-2} N / P W M, \quad \eta_{2}=9.69 \cdot 10^{-3} N / P W M$, $\eta_{3}=5.11 \cdot 10^{-3} N / P W M, \quad \eta_{4}=8.37 \cdot 10^{-3} N / P W M \quad$ and $\hat{\eta}=1 \cdot 10^{-2} N / P W M$. In this linear case, one can state that:

$\mathbf{F}_{B}=\mathbf{A} \cdot \mathscr{M} \cdot \widehat{\mathscr{M}}^{-1} \cdot\left[\mathbf{A}^{+} \mathbf{M}_{m}\right] \cdot\left[\begin{array}{l}\mathbf{F}_{B}^{d} \\ r_{m}\end{array}\right]$

The computation of the $Q$ matrix is done according to the previously described protocol, and ends with the curves exposed at Fig. 13-center, where it is clear that in the range where no actuator saturates, the corrective coefficients are constant. This can be easily explained considering the linearity of the characteristics. The use of the matrix $\mathbb{Q}$ is done according to Equation (34).

$\mathbf{F}_{B}=\mathbf{A} \cdot \mathscr{M} \cdot Q \vec{Q} \cdot \widehat{\mathscr{M}}^{-1} \cdot\left[\mathbf{A}^{+} \mathbf{M}_{m}\right] \cdot\left[\begin{array}{l}\mathbf{F}_{B}^{d} \\ r_{m}\end{array}\right]$

Since each trials (for a given $c_{0}$ ) results in a stable behavior of the system (velocities are null), the resulting forces from the actuation to the system is null. In other words, in this stabilized situation, each mo-

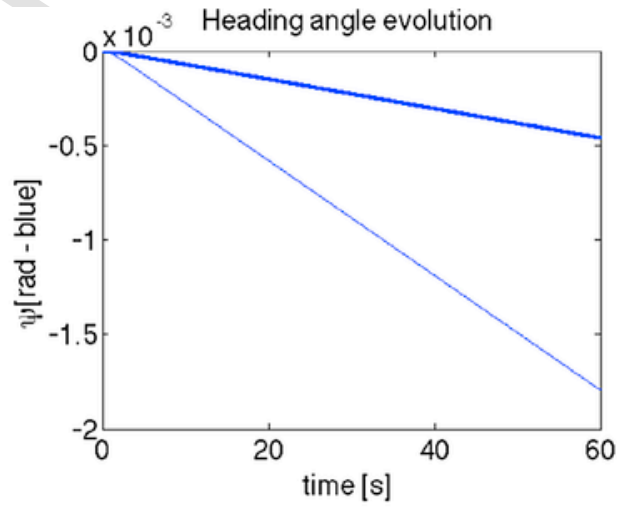

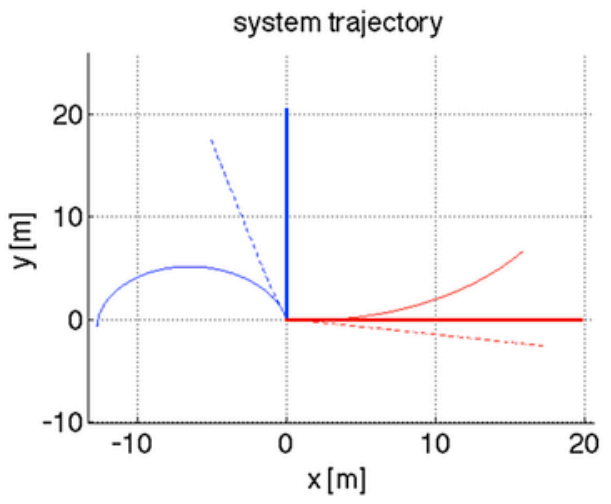

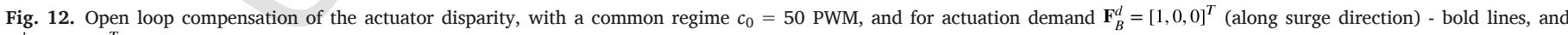

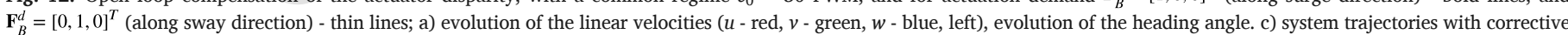

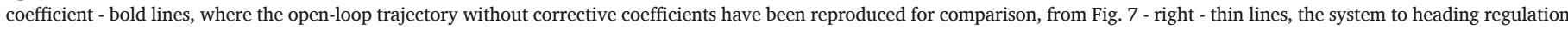

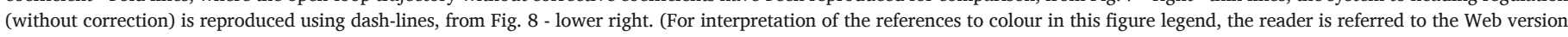
of this article.) 


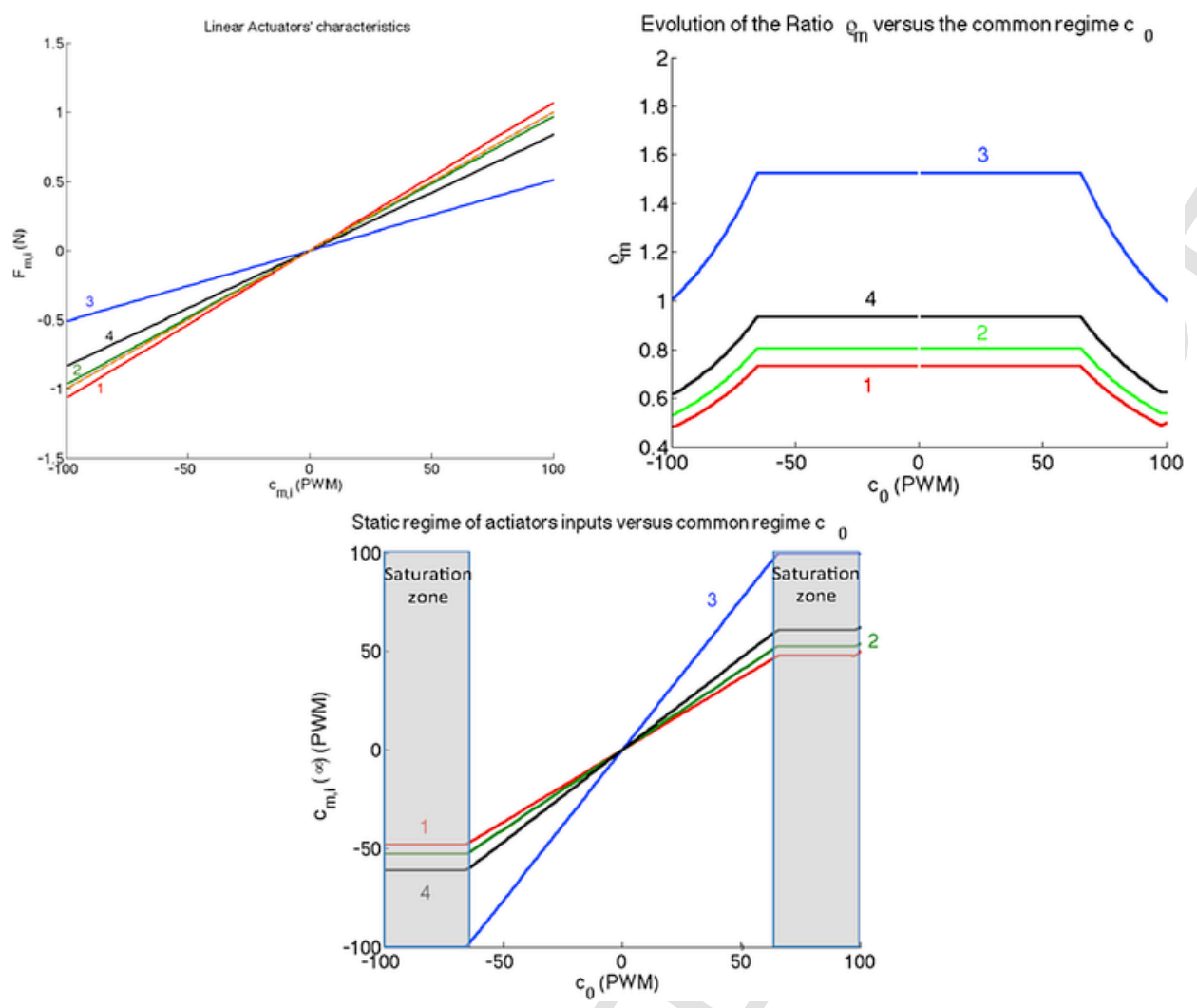

Fig. 13. Linear characteristics of the actuators (left), corrective coefficients (center), static inputs of the actuators (right).

tor produces the same force, as Equation (35) expresses.

$$
\begin{aligned}
\forall c_{0}, \eta_{1} \cdot c_{m, 1}^{\infty}\left(c_{0}\right) & =\eta_{2} \cdot c_{m, 2}^{\infty}\left(c_{0}\right) \\
& =\eta_{3} \cdot c_{m, 3}^{\infty}\left(c_{0}\right) \\
& =\eta_{4} \cdot c_{m, 4}^{\infty}\left(c_{0}\right)
\end{aligned}
$$

Dividing the last relation by $c_{0}$ yields:

$$
\eta_{1} \cdot \alpha_{1}=\eta_{2} \cdot \alpha_{1}=\eta_{3} \cdot \alpha_{1}=\eta_{4} \cdot \alpha_{1}
$$

Expanding the relation $\mathscr{M} \cdot \mathbb{Q} \cdot \widehat{\mathscr{M}}^{-1}$ yields:

$$
\mathscr{M} \cdot \mathbb{Q} \cdot \widehat{\mathscr{M}}^{-1}=\left[\begin{array}{cccc}
\frac{\eta_{1}}{\eta_{0}} \cdot \alpha_{1} & 0 & 0 & 0 \\
0 & \frac{\eta_{2}}{\eta_{0}} \cdot \alpha_{2} & 0 & 0 \\
0 & 0 & \frac{\eta_{3}}{\eta_{0}} \cdot \alpha_{3} & 0 \\
0 & 0 & 0 & \frac{\eta_{4}}{\eta_{0}} \cdot \alpha_{4}
\end{array}\right]
$$

$\mathscr{M} \cdot Q \cdot \widehat{\mathscr{M}}^{-1}=\frac{\eta_{1}}{\eta_{0}} \cdot \alpha_{1} \cdot \rrbracket_{4}=k_{\mathscr{Q}} \cdot \rrbracket_{4}$

Note that the quantity $\frac{\eta_{1}}{\eta_{0}} \cdot \alpha_{1}$ is unknown but constant. Hence, the relation (34) is rewritten as:

$$
\mathbf{F}_{B}=\frac{\eta_{1}}{\eta_{0}} \cdot \alpha_{1} \cdot \mathbf{A} \cdot\left[\mathbf{A}^{+} \mathbf{M}_{m}\right] \cdot\left[\begin{array}{c}
\mathbf{F}_{B}^{d} \\
r_{m}
\end{array}\right]=\frac{\eta_{1}}{\eta_{0}} \cdot \alpha_{1} \cdot \mathbf{F}_{B}^{d}
$$

$$
\mathbf{F}_{B}=k_{\mathscr{Q}} \cdot \mathbf{F}_{B}^{d}
$$

which explains de decoupling effect, in the linear case, if singular values of $\mathbf{A}$ are non null and the scalar $k_{\mathscr{Q}}$ is considered as one static gain.

We can now state the following proposition:

\section{Proposition}

Consider actuation system of Figure 2, composed with 4 thrusters mounted with a vectorial configuration with characteristics of Equation (18). These 4 actuators provide 4 individual thrusts denoted with the $(4 \times 1)$ vector $\mathbf{F}_{m}$ (cf. Eq. (6)), and are controlled with the $(4 \times 1)$ (PWM) input vector $\mathbf{c}_{m}$ (cf. Eq. (5)). The linear motor characteristic is denoted with the matrix $\mathrm{M}$, diagonally composed with the static (and unknown) gain of each actuator, providing the relation $\mathbf{F}_{m}=\mathbf{M} \cdot \mathbf{c}_{m}$ (cf. Eq (32)). Consider that an estimation of this characteristic is available, with the form $\widehat{\mathbb{M}}=\operatorname{diag}(\hat{\eta})$, where $\hat{\eta}$ is a common estimated gain of the actuators (cf. Eq (31)).

The resulting actuation, expressed in the body frame is denoted $\mathbf{F}_{B}=\mathbf{A} \cdot \mathbf{F}_{m}$ (cf. Eq. (9)), where $\mathbf{A}$ denotes the $(3 \times 4)$ actuation matrix expressed at Equation (19).

The closed-loop control of Equation (25) and (26), with a common regime $r_{m} \neq 0$ and where the matrix $\mathbf{M}_{m}$ is chosen as $\mathbf{M}_{m} \in k e r(A)$ (cf Eq (20)), results in the system stabilization around null velocities, providing a set of actuation inputs $\mathbf{c}_{m}^{\infty}$. Then the use of $\mathbb{Q}=\operatorname{diag}\left(c_{m, i}^{\infty} / c_{0}\right)$ matrix (cf. Eq (27)) in the open-loop control expression $\mathbf{c}_{m}=\mathscr{Q} \cdot \widehat{\mathscr{M}}^{-1} \cdot\left[\mathbf{A}^{+} \mathbf{M}_{m}\right] \cdot\left[\begin{array}{l}\mathbf{F}_{B}^{d} \\ r_{m}\end{array}\right]$ (cf. Eq (30)) provides the decoupled relation $\mathbf{F}_{B}=k_{\mathscr{Q}} \cdot \mathbf{F}_{B}^{d}$ (cf. Eq (40)), where $k_{\mathbb{Q}}$ is a static and scalar gain.

Proof 
The use of closed-loop control expression (25) and (26) provides a stable static behavior, such that:

$\mathbf{F}_{B}^{\infty}=\mathbf{A} \cdot \mathscr{M} \cdot \mathbf{c}_{m}^{\infty}=0$

Note that the choice (28) for matrix $\mathbb{Q}$ respects the following relation $\mathbf{c}_{m}^{\infty}=Q \cdot \widehat{\mathscr{M}}^{-1} \cdot \mathbf{M}_{m}$

Then considering the two last expression yields:

$$
\mathbf{A} \cdot \mathscr{M} \cdot Q \cdot \widehat{\mathscr{M}}^{-1} \cdot \mathbf{M}_{m}=0
$$

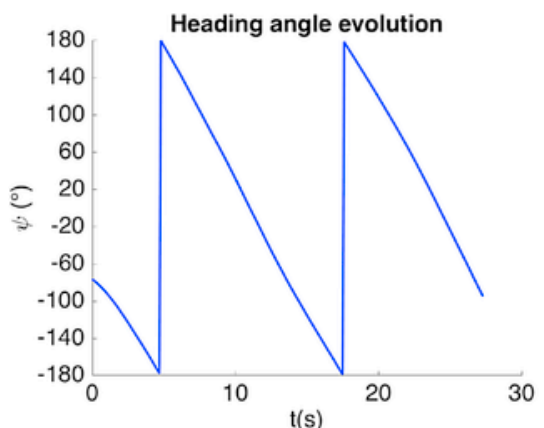

$t(s)$
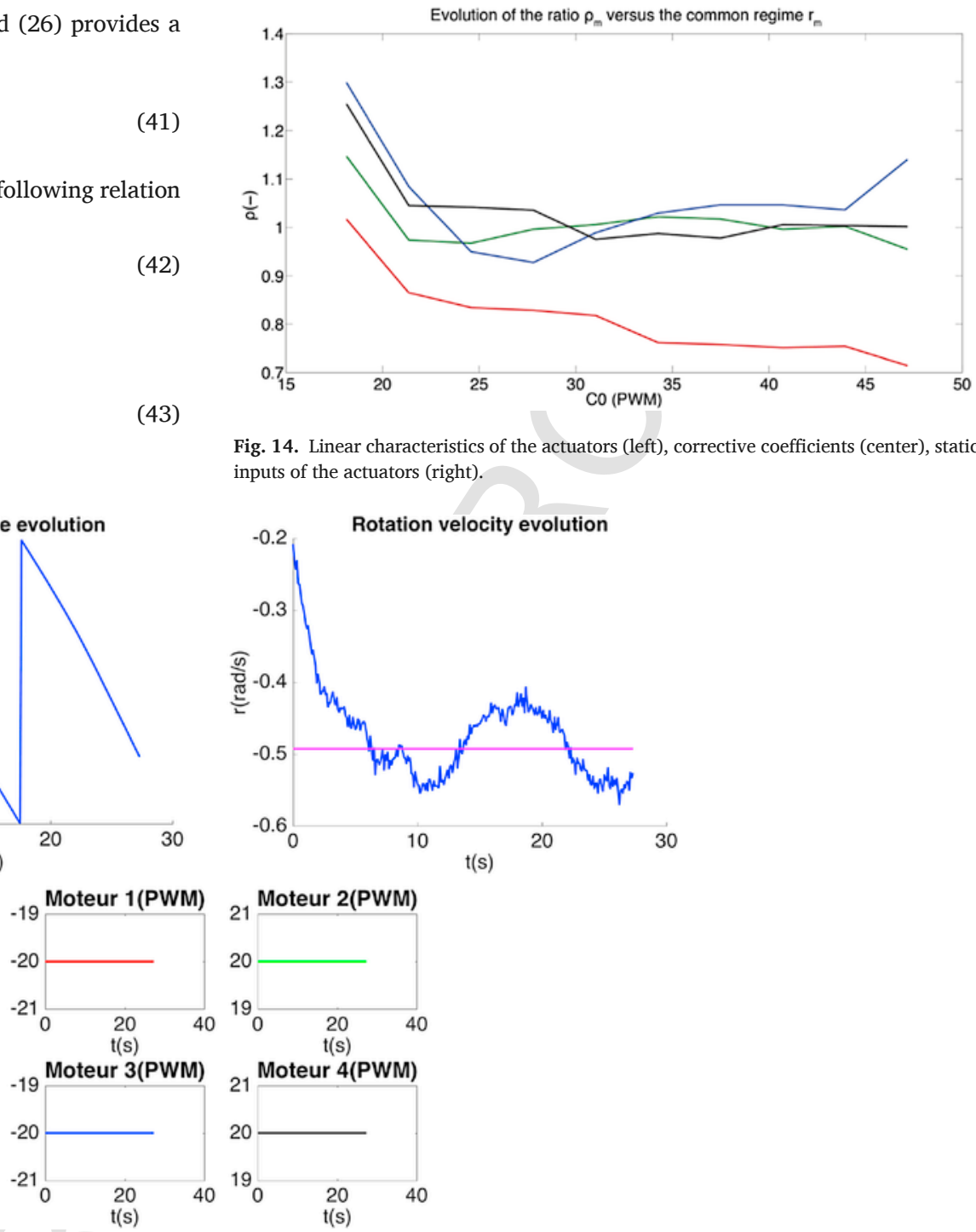

Fig. 14. Linear characteristics of the actuators (left), corrective coefficients (center), static inputs of the actuators (right).
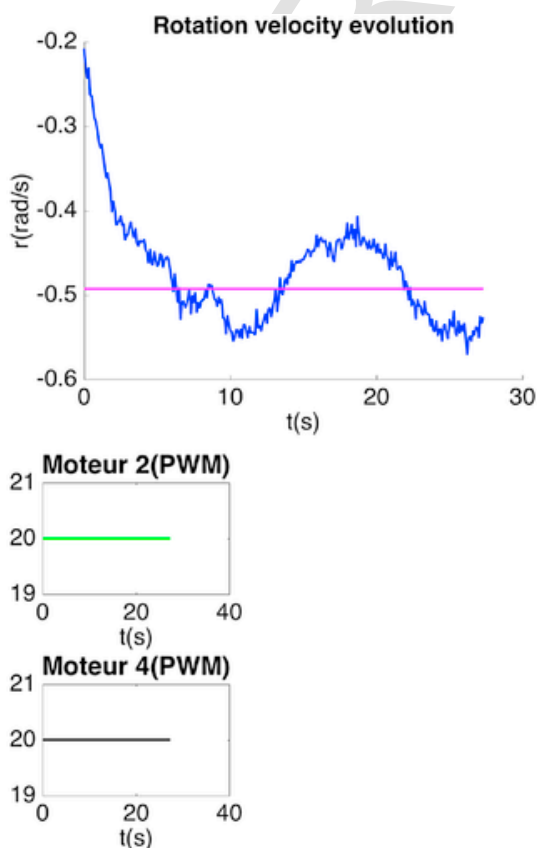

Fig. 15. Curves without correction coefficients: Heading angle evolution (left), rotation velocity evolution (center), inputs of the actuators (right).

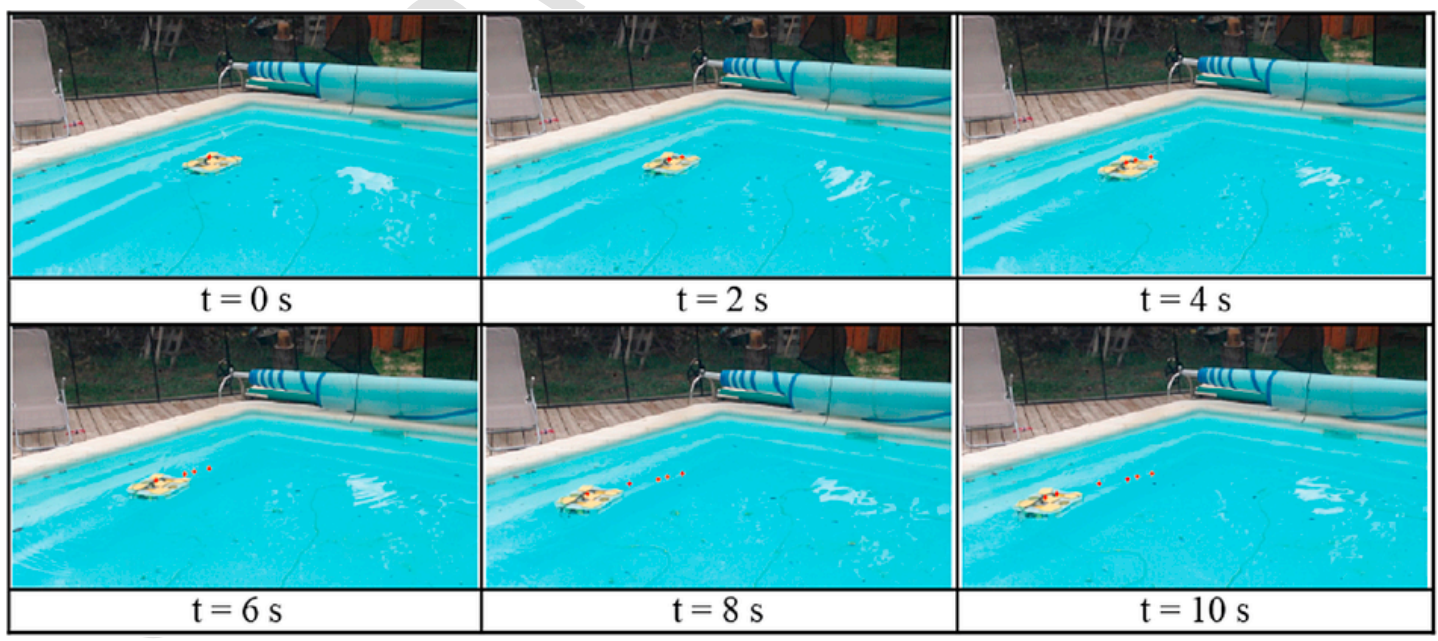

Fig. 16. Unwanted effect with a basic PID yaw controller. 

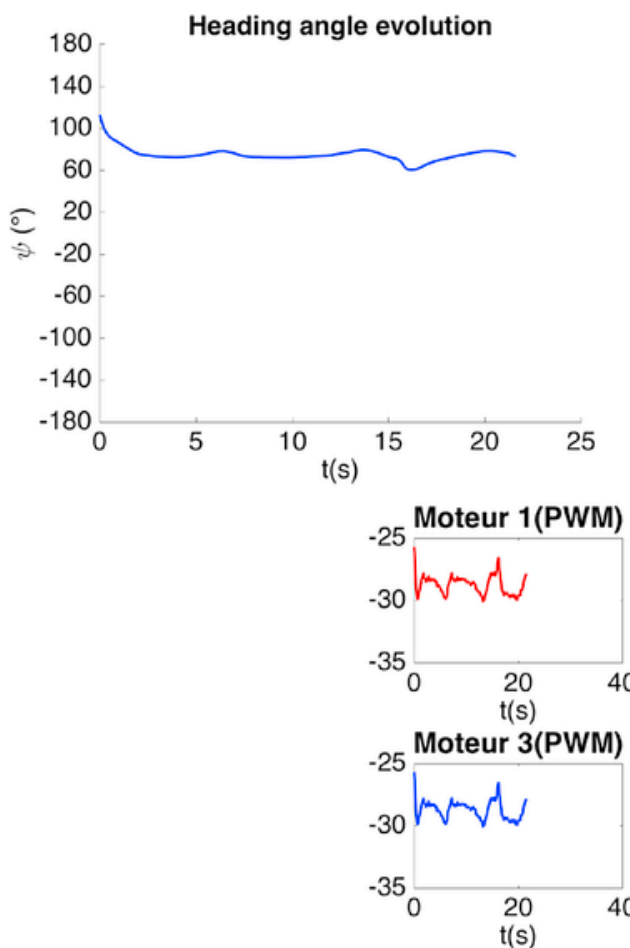

Fig. 17. Curves without correction coefficients with a basic PID yaw controller: Heading angle evolution (left), rotation velocity evolution (center), inputs of the actuators (right).
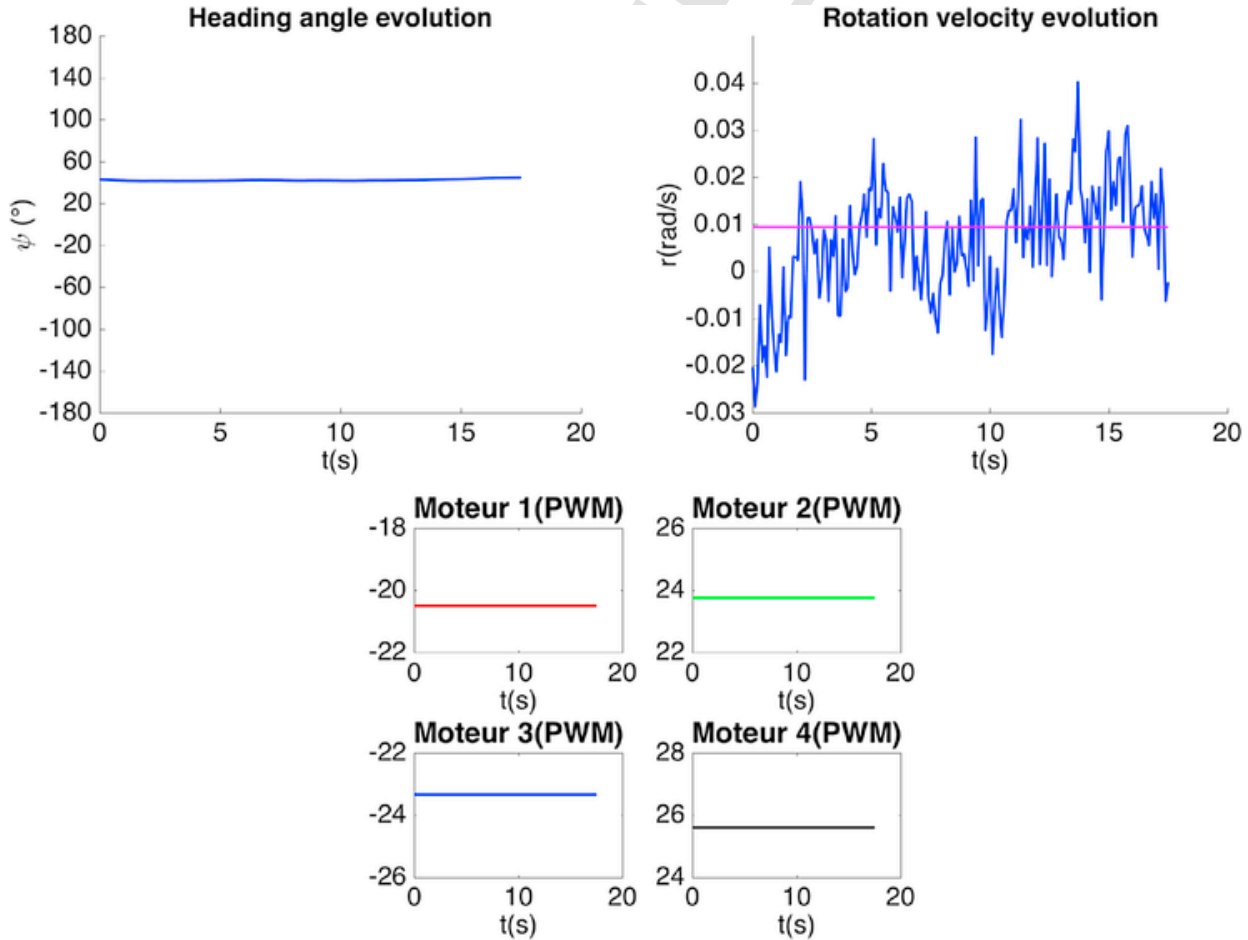

Fig. 18. Curves with correction coefficients: Heading angle evolution (left), rotation velocity evolution (center), inputs of the actuators (right). 


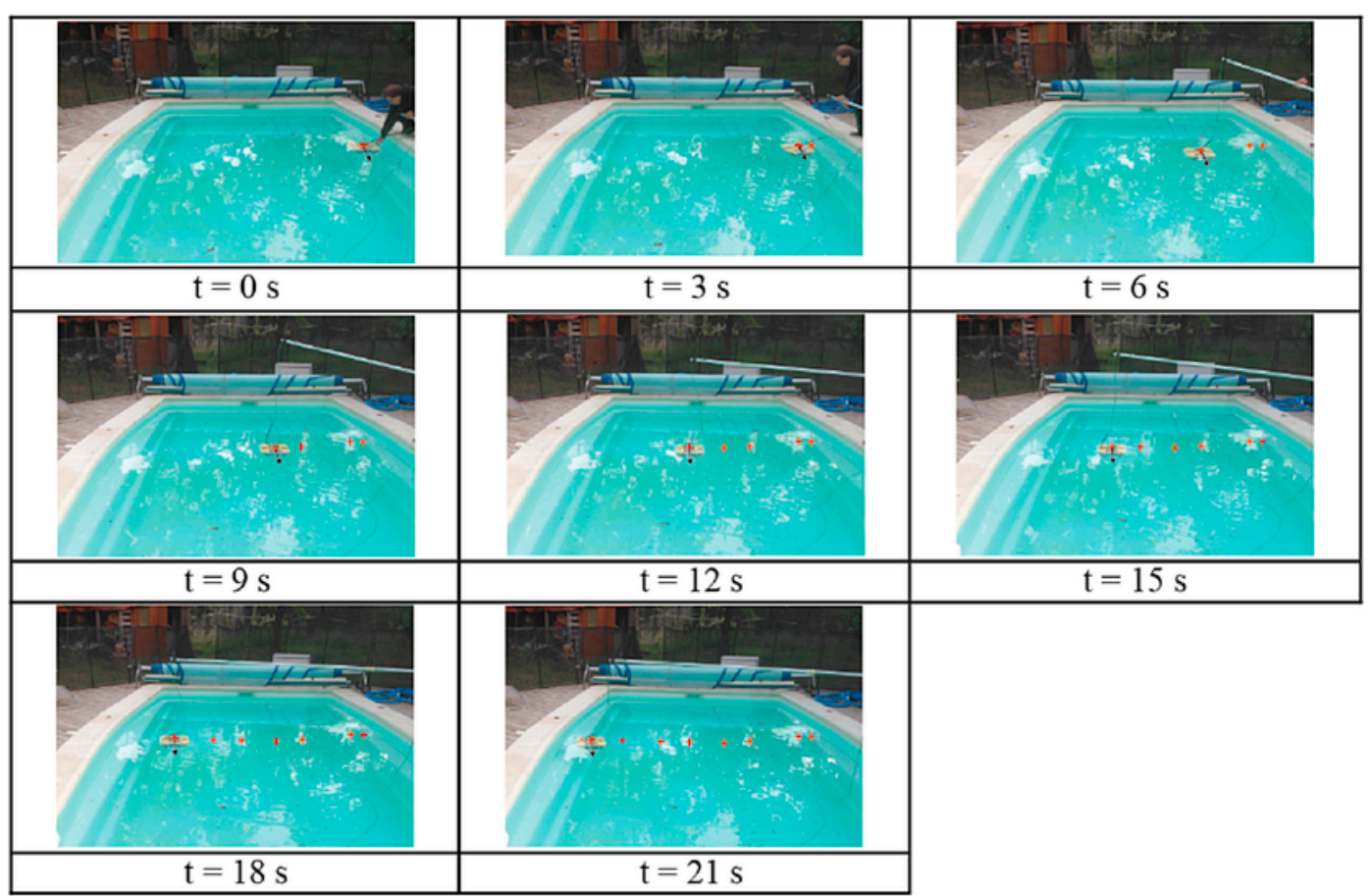

Fig. 19. Decoupling effect in open loop with $\mathbf{F}_{B}^{d}=[0,4,0]$.

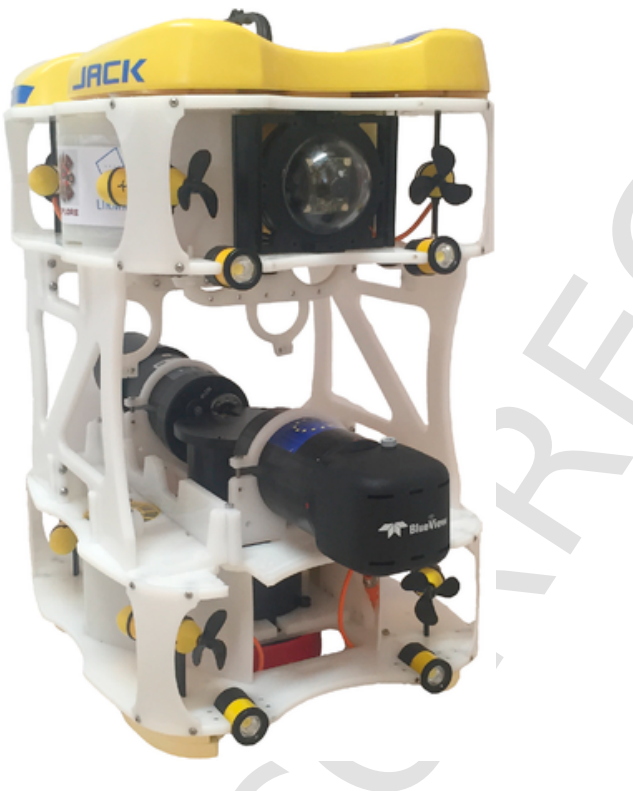

Fig. 20. The Ulysse system.

Then the actuation resulting force can be written as:

$$
\begin{aligned}
\mathbf{F}_{B} & =\mathbf{A} \cdot \mathscr{M} \cdot \mathbb{Q} \cdot \widehat{\mathscr{M}}^{-1} \cdot\left[\mathbf{A}^{+} \mathbf{M}_{m}\right] \cdot\left[\begin{array}{l}
\mathbf{F}_{B}^{d} \\
r_{m}
\end{array}\right] \\
& =\mathbf{A} \cdot \mathscr{M} \cdot \mathbb{Q} \cdot \widehat{\mathbb{M}}^{-1} \cdot \mathbf{A}^{+} \cdot \mathbf{F}_{B}^{d}
\end{aligned}
$$

Let $\mathbf{B}=\mathscr{M} \cdot \mathcal{Q} \cdot \widehat{\mathbb{M}}^{-1}$, then one can state that $\mathbf{M}_{m} \in \operatorname{ker}(\mathbf{A})$ (by construction) and $\mathbf{M}_{m} \in \operatorname{ker}(\mathbf{A} \cdot \mathbf{B})$ (since 43), or equivalently, $\mathbf{A} \cdot \mathbf{B} \cdot \mathbf{M}_{m}=0$ , which implies that $\mathbf{B} \cdot \mathbf{M}_{m} \in \operatorname{ker}(\mathbf{A})$. Since in our case, $\mathbf{M}_{m}$ and $\mathbf{B} \cdot \mathbf{M}_{m}$ are $(4 \times 1)$, they are necessarily collinear, since previous argument. Hence following relation can be written: $\mathbf{B} \cdot \mathbf{M}_{m}=k_{\mathscr{Q}} \cdot \mathbf{M}_{m}$. Note that $\mathbf{B}$ is composed with diagonal matrices, and is diagonal itself. Then, since the form of $\mathbf{M}_{m}$ (cf. Eq. (20)), one can conclude that $\mathbf{B}=k_{\mathbb{Q}} \cdot \mathbb{\nabla}_{4}$, where $\mathbb{\square}_{4}$ stands for the $(4 \times 4)$ identity matrix. Hence relation (44) can be written as

$$
\begin{aligned}
& \mathbf{F}_{B}=\mathbf{A} \cdot \mathscr{M} \cdot Q \cdot \widehat{\mathscr{M}}^{-1} \cdot \mathbf{A}^{+} \cdot \mathbf{F}_{B}^{d} \\
& =\mathbf{A} \cdot k_{\mathscr{Q}} \cdot \mathbb{q}_{4} \cdot \mathbf{A}^{+} \cdot \mathbf{F}_{B}^{d} \\
& =k_{\mathscr{Q}} \cdot \mathbf{A} \cdot \mathbf{A}^{+} \cdot \mathbf{F}_{B}^{d} \\
& =\quad k_{\mathscr{Q}} \cdot \mathbf{F}_{B}^{d}
\end{aligned}
$$

since the argumentation following Equation (12). This ends the proof.

\section{Experimental validation}

We experimentally validate this approach using the Jack system, with a heading regulation involving the IMU measurement, providing the third component of $\mathbf{F}_{B}^{d}, \Gamma_{r}$. The linear velocity regulation is visually done in our test tank. The operator manipulates a joystick, according to the observed linear movements, and directly provides the two first components of $\mathbf{F}_{B}^{d}, F_{u}$ and $F_{v}$. We proceed to the experimentation consisting in providing to the system different common regimes $c_{0}$ and, for each of them, realize the control action $\mathbf{F}_{B}^{d}$ (with IMU and visual observation) which provides a steady system behavior $(u, v, r=0)$, according to (17). This ends up with an identification of the steady values $c_{m, i}^{\infty}$, corrective coefficients $\alpha_{i}\left(c_{0}\right)$ is computed according to (27).

We have restricted the evolution of $c_{0}$ from 20 to 50 PWM. Indeed the Jack's actuators exhibit a poor negative response. Hence, we restrict the range of accessible motor regime to positive. Moreover, we stopped the experiments when we notice that one motor reaches its saturation limit.

As we can see in Fig. 14, the effect of actuators disparity is important. It induces a rotational movement with velocity $r$ shown in blue at Fig. 15-center. The experimentation has been stopped after $27 \mathrm{~s}$ because the effect of the cable was no more negligible after three complete revolutions, as shown at Fig. 15-left. We extract form these curves 
an estimation of the steady state the system should asymptotically reach, purple line of 15-center.

Second experiment consists in closing the heading regulation loop on the IMU measurements with a classic PID controller. As Fig. 17 shows, heading is stabilized. But, snapshots of Fig. 16 clearly indicate an undesired linear movement along surge and sway ( $u$ and $v$ ).

The use of the correction matrix $Q$, according to (30) provides the open loop system's response of Fig. 18, where improvement is clear, and for which a common regime of $c_{0}=24$ PWM has been chosen.

Fig. 19, shows the disparity compensation for a desired effort of $\mathbf{F}_{B}^{d}=[0,40]^{T}$, in open loop. The decoupling effect is clear.

\section{Conclusion and future work}

The actuation system of an underwater robotic system impacts global system reactivity. The use of a redundant actuation allows for compensating some drawbacks, as dead-zone effects, as shown in Ropars et al. (2015). Nevertheless, redundant actuation, as the vectorial configuration of many underwater robots, brings the question of the thrusters' disparity effects. While the undesired effect on rotational movement can be easily compensated using a closed loop yaw regulation using an IMU system, the linear undesired effects are more difficult to compensate. This requires the use of a velocity sensor, e.g. Doppler Velocity Log, which is not appropriate to low cost and small size underwater robot. We propose to perform preliminary closed loop tests on the system in order to identify a correction matrix $\mathbb{Q}$, which reduces the not desired effects of actuators disparity, in open loop. The rationale of the use of this correction matrix $\mathbb{Q}$ is formally demonstrated in the linear case. The performances of this solution is then experimentally shown on the robot system Jack.

The next step is to generalize our approach to our new demonstrator, the underwater robot Ulysse depicted at Fig. 20, along the 6 degrees of freedom. It carries 12 thrusters that allows for a larger redundancy space.

\section{Acknowledgments}

The authors would like to warmly thank S. Louis for his constant help on this study, the French labex NUMEV, and the FEDER action for supporting this research.

\section{References}

Avila, J., Adamowski, J., Maruyama, N., Takase, F., Saito, M., 2012. Modeling and identification of an open-frame underwater vehicle: the yaw motion dynamics. J. Intell. Rob. Syst. 66 (1-2), 37-56. https://doi.org/10.1007/s10846-011-9625-x https://doi. org/10.1007/s10846-011-9625-x.

Bessa, W.M., Dutra, M.S., Kreuzer, E., 2013. Dynamic positioning of underwater robotic vehicles with thruster dynamics compensation. Int. J. Adv. Rob. Syst. 10, 1. https: //doi.org/10.5772/56601 http://www.intechopen.com/journals/international \}ournal \{_\}of \{ \}advanced\{_\}robotic\{_\}systems/

dynamic-positioning-of-underwater-robotic-vehicles-with-thruster-dynamics-compensation.

Choi, S., Takashige, G., Yuh, J., 1994. Experimental study on an underwater robotic vehicle: ODIN. In: Proceedings of IEEE Symposium on Autonomous Underwater Vehicle Technology (AUV'94). IEEE, Cambridge, pp. 79-84. https://doi.org/10.1109/ AUV.1994.518610 htm?arnumber $=518610$.

http://ieeexplore.ieee.org/lpdocs/epic03/wrapper.

Chyba, M., Haberkorn, T., Smith, R.N., Choi, S.K, Mariani, G., McLeod, C., 2008. Efficient control of an autonomous underwater vehicle while accounting for thruster failure. In: International Conference on Computer Applications and Information Technology in the Maritime Industries, COMPIT '08, no. 2, Liege

Flacco, F., De Luca, A., Khatib, O., 2012. Motion control of redundant robots under joint constraints: saturation in the null space. In: International Conference on Robotics and Automation. pp. 285-292.

Fossen, T.I., 1994. Guidance and Control of Ocean Vehicles. John Wiley \& Sons Ltd., doi:0471941131 http://www.amazon.com/Thor-I.-Fossen/e/B001HQ3UVM.
Fuqiang, L., Demin, X., Jian, G., Rongxin, C., 2013. Fault tolerant control for an autonomous underwater vehicle to dock with thruster redundancy. In: Chinese Control Conference (CCC). IEEE, Xi'an, pp. 6186-6190.

Garus, J., 2004. Optimization of thrust allocation in the propulsion system of an underwater vehicle. Int. J. Appl. Math. Comput. Sci. 14 (4), 461-467, doi:10.1.1.385.4601.

Golub, G.H., Van Loan, C.F., 1957. Matrix computations. Phys. Today 10 (8), 48. https:// doi.org/10.1063/1.3060478 http://www.ncbi.nlm.nih.gov/pubmed/18273219 http: //scitation.aip.org/content/aip/magazine/physicstoday/article/10/8/10.1063/1. 3060478.

Hanai, A., Choi, H.T., Choi, S.K., Yuh, J., 2003. Minimum energy based fine motion control of underwater robots in the presence of thruster nonlinearity. In: International Conference on Intelligent Robots and Systems, IROS '03, vol. 1, IEEE, Las Vegas, pp. 559-564. https://doi.org/10.1109/IROS.2003.1250688, vol. 1.

Hanai, A., Rosa, K., Choi, S., Yuh, J., 2004. Experimental analysis and implementation of redundant thrusters for underwater robots. In: International Conference on Intelligent Robots and Systems, IROS '04, vol. 2, IEEE, Sendei, pp. 1109-1114. https://doi.org/ 10.1109/IROS.2004.1389545 http://ieeexplore.ieee.org/lpdocs/epic03/wrapper. htm?arnumber $=1389545$.

Horn, R.A., Johnson, C.R., 1985. Matrix Anal. 169, https://doi.org/10.1002/zamm. 19870670330.

Indiveri, G., 1998. Modelling and Identification of Underwater Robotic Systems. Ph.D. thesis University of Genova, Genova.

Johansen, T. a., Fossen, T.I., 2013. Control allocation - a survey. Automatica 49 (5), 1087-1103. https://doi.org/10.1016/j.automatica.2013.01.035 https://doi.org/10. 1016/j.automatica.2013.01.035.

Khalil, W., Dombre, E., Nagurka, M., 2003. Modeling, identification and control of robots. Appl. Mech. Rev. 56 (3), B37. https://doi.org/10.1115/1.1566397

Kim, Jinhyun, Shon, Woong Hee, Lee, Ho-Gil, Chung, Wan Kyun, 2006. Preliminary thruster control experiments for underwater vehicle positioning. In: International Conference on Robotics and Automation, 2006. ICRA '06., no. May. IEEE, Orlando, pp. 3233-3237. https://doi.org/10.1109/ROBOT.2006.1642194 http://ieeexplore.ieee org/lpdocs/epic03/wrapper.htm?arnumber $=1642194$.

Liang, X., Zhang, J., Qin, Y., Yang, H., 2013. Dynamic modeling and computer simulation for autonomous underwater vehicles with fins. J. Comput. 8 (4), 1058-1064. https: //doi.org/10.4304/jcp.8.4.1058-1064 http://ojs.academypublisher.com/index.php/ jcp/article/view/7811.

Liégeois, A., 1977. Automatic supervisory control of the configuration and behavior of multibody mechanisms. IEEE Trans. Syst. Man Cybern. 7 (12), 868-871. https: //doi.org/10.1109/TSMC.1977.4309644 http://ieeexplore.ieee.org/lpdocs/epic03/ wrapper.htm?arnumber $=4309644$.

Low, K.H., 2009. Modelling and parametric study of modular undulating fin rays for fish robots. Mech. Mach. Theor. 44, 615-632. https://doi.org/10.1016/j. mechmachtheory.2008.11.009.

Mansard, N., 2006. Enchainement de taches robotiques. Ph.D. thesis Université de Rennes 1 , Rennes.

Nakajoh, H., Osawa, H., Miyazaki, T., Hirata, K., Sawa, T., Utsugi, H., 2012. Development of work class ROV applied for submarine resource exploration in JAMSTEC. In: Oceans 2012. IEEE, Yeosu, https://doi.org/10.1109/OCEANS-Yeosu.2012.6263437.

Nakamura, Y., Hanafusa, H., Yoshikawa, T., 1987. Task-priority based redundancy control of robot manipulators. Int. J. Robot Res. 6 (2), 3-15. https://doi.org/10.1177/ 027836498700600201 http://ijr.sagepub.com/cgi/content/long/6/2/3.

Omerdic, E., Roberts, G., 2004. Thruster fault diagnosis and accommodation for open-frame underwater vehicles. Contr. Eng. Pract. 12 (12 SPEC. ISS.), 1575-1598. https://doi.org/10.1016/j.conengprac.2003.12.014 http://linkinghub.elsevier.com/ retrieve/pii/S0967066103002892.

Pivano, L., 2008. Thrust Estimation and Control of Marine Propellers in Four- Quadrant Operations. no. April NTNU.

Ridao, P., Tiano, A., El-Fakdi, A., Carreras, M., Zirilli, A., 2004. On the identification of non-linear models of unmanned underwater vehicles. Contr. Eng. Pract. 12 (12 SPEC. ISS.), 1483-1499. https://doi.org/10.1016/j.conengprac.2004.01.004 http:// linkinghub.elsevier.com/retrieve/pii/S0967066104000152.

Ropars, B., Lasbouygues, A., Lapierre, L., Andreu, D., 2015. Thruster s dead - zones compensation for the actuation system of an underwater vehicle. In: Control Conference (ECC), 2015 European. IEEE, Linz, Austria.

Yang, R., Clement, B., Mansour, A., Li, H.J., Li, M., Wu, N.L., 2014. Modeling of a complex-shaped underwater vehicle. In: 2014 IEEE International Conference on Autonomous Robot Systems and Competitions (ICARSC), no. L. IEEE, Espinho, pp. 36-41. https://doi.org/10.1109/ICARSC.2014.6849759 https://www.researchgate.net/ publication/273950011\{_\}Robust \{_\}Control\{_\}Application \{_\}To\{_\}Ciscrea \{_ \}Underwater\{_\}Vehicle.

Yang, R., Clement, B., Mansour, A., Li, H.J., Li, M., 2015. Invited paper: robust control application to ciscrea underwater vehicle. In: OCEANS'15/MTS/IEEE. IEEE, Genova https://www.researchgate.net/publication/273950011_Robust_Control_Application_ To_Ciscrea_Underwater_Vehicle.

Yoerger, D., Cooke, J., Slotine, J.-J., 1990. The influence of thruster dynamics on underwater vehicle behavior and their incorporation into control system design. IEEE J. Ocean. Eng. 15 (3), 167-178. https://doi.org/10.1109/48.107145, URL http://ieeexplore.ieee.org/ielx1/48/3268/00107145.pdf?tp $=\{\&\}$ arnumber $=107145\{\&\}$ isnum ber $=3268 \$ \backslash$ delimiter"026E30F\$nhttp://ieeexplore.ieee.org/xpls/abs\{_\}all.jsp?arnumber $=107145$. 